Aus der Abteilung Gynäkologie und Geburtshilfe

(Prof. Dr. med. G. Emons)

im Zentrum Frauenheilkunde

der Medizinischen Fakultät der Universität Göttingen

\title{
Sonographische Softmarker
}

Wertvolle Screeningparameter in der Pränataldiagnostik zur Detektion fetaler Chromosomenanomalien

\author{
INAUGURAL - DISSERTATION \\ zur Erlangung des Doktorgrades \\ der Medizinischen Fakultät der \\ Georg-August-Universität zu Göttingen
}

vorgelegt von

ANNA JANINA KNAUER

aus

Detmold

Göttingen 2009 
Dekan:

I. Berichterstatter:

II. Berichterstatter/in:

III. Berichterstatter/in:

Tag der mündlichen Prüfung:
Prof. Dr. med. C. Frömmel

PD Dr. med. H.-U. Pauer 


\section{Inhaltsverzeichnis}

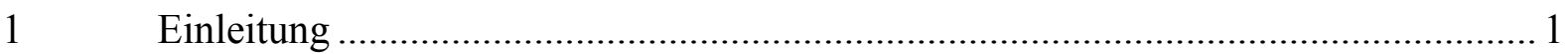

1.1 Methoden und Indikationen der Pränataldiagnostik.............................................. 1

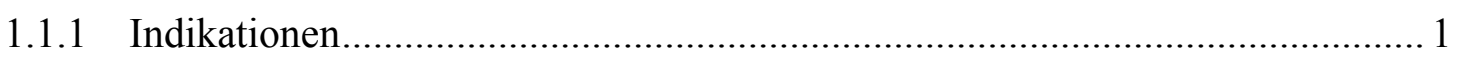

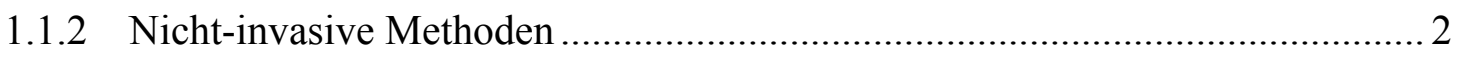

1.1.2.1 Das humangenetische Beratungsgespräch .......................................... 2

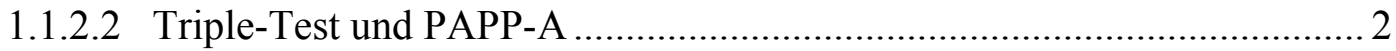

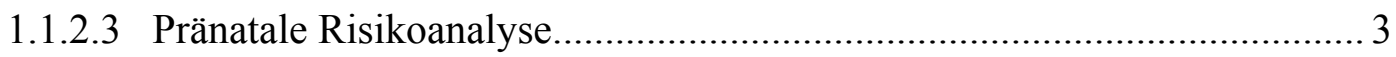

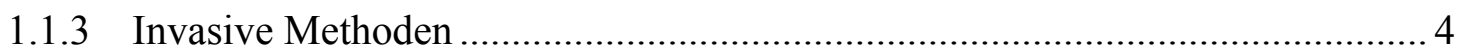

1.1.3.1 Amniozentese, Chorionzottenbiopsie und Chordozentese ....................... 4

1.1.3.2 Risiken der invasiven Pränataldiagnosik................................................ 5

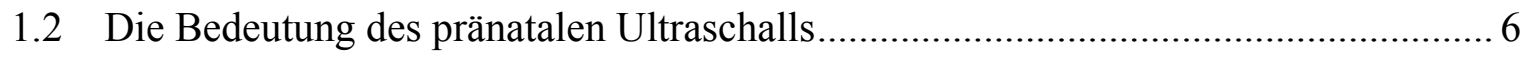

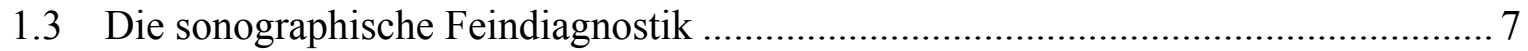

1.4 Sonographische Softmarker im pränatalen Ultraschall ........................................... 9

1.5 Definition und Diagnostik sonographischer Softmarker....................................... 11

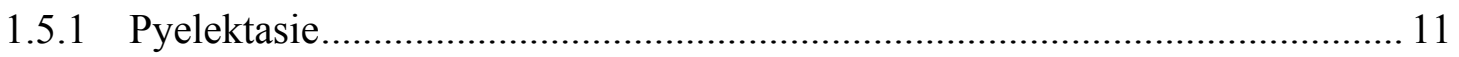

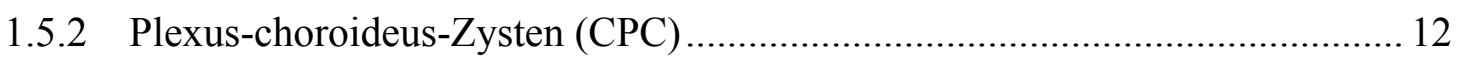

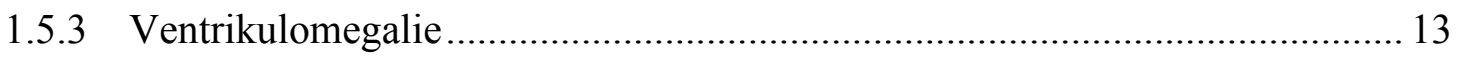

1.5.4 Kurzes Femur / kurzer Humerus …............................................................. 13

1.5.5 Intrakardialer echogener Fokus (ICEF) .................................................. 14

1.5.6 Singuläre Nabelschnurarterie (SUA) ……................................................ 15

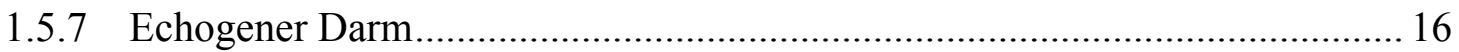

1.5.8 Sonderfall Nackenödem / erhöhte Nackentransparenz (NT) ......................... 16

1.6 Sonographische Softmarker und fetale Chromosomenanomalien ........................... 18

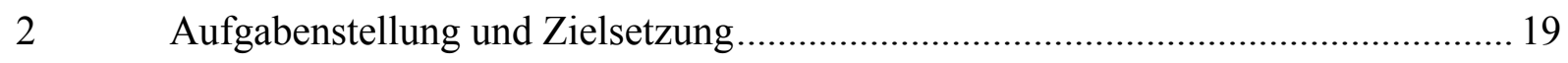

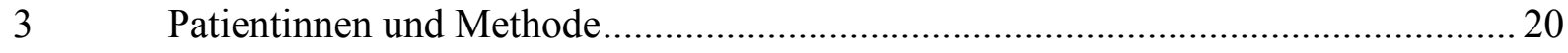

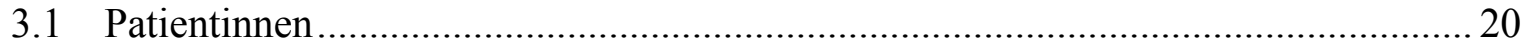

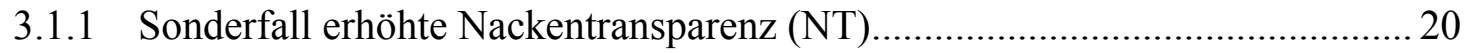

3.1.2 Vergleichskollektiv der Feten mit echten Fehlbildungen und anderen

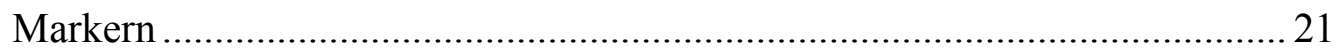

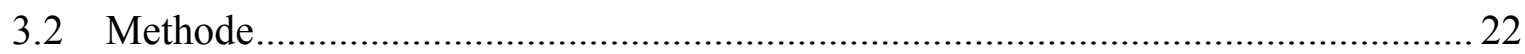


4.1 Altersverteilung der Patientinnen und Gestationswoche (SSW) zum Zeitpunkt der Amniozentese

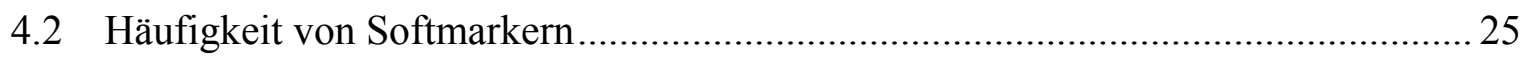

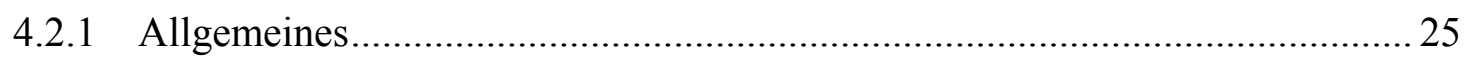

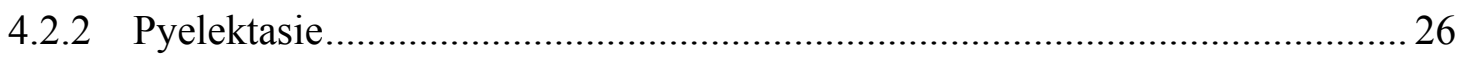

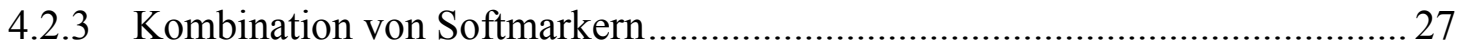

4.3 Häufigkeit von fetalen Fehlbildungen und anderen Markern ................................. 28

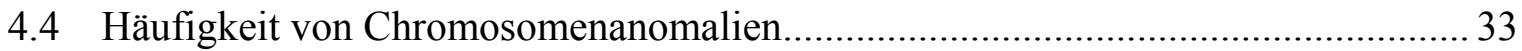

4.5 Häufigkeit von Chromosomenanomalien bei positivem Softmarkerbefund,

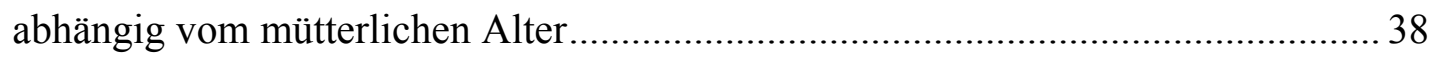

4.6 Häufigkeit von Chromosomenanomalien bei Vorliegen eines isolierten

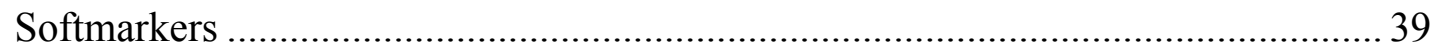

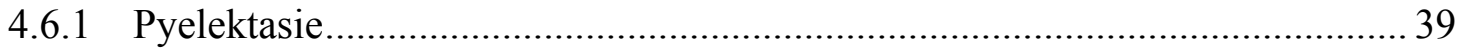

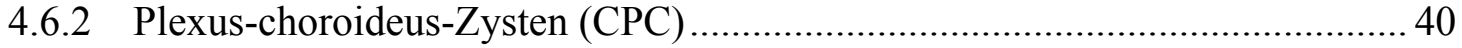

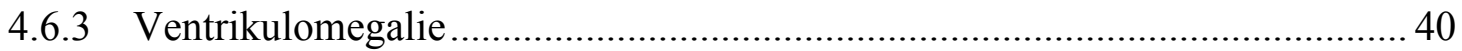

4.6.4 Kurzes Femur / kurzer Humerus ................................................................... 40

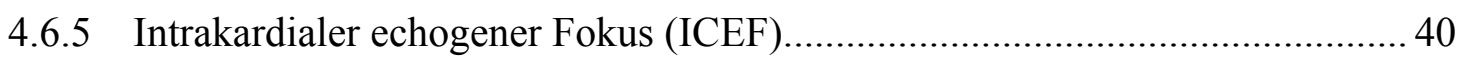

4.6.6 Singuläre Nabelschnurarterie (SUA) ....................................................... 41

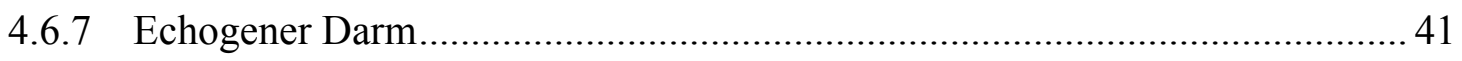

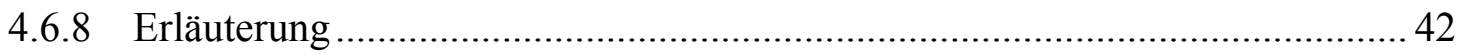

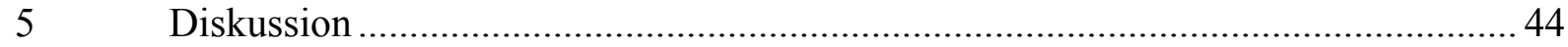

5.1 Assoziation von sonographischen Softmarkern mit fetalen

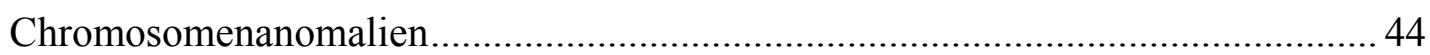

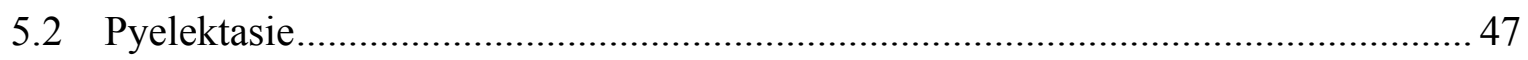

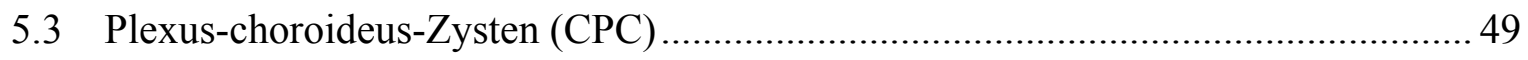

5.4 Ventrikulomegalie, intrakardialer echogener Fokus (ICEF), singuläre Nabelschnurarterie (SUA), echogener Darm und kurzes Femur / kurzer Humerus ............50 50

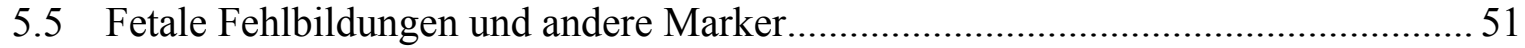

5.6 Bedeutung der sonographischen Softmarker für das pränatale Screening ................ 53

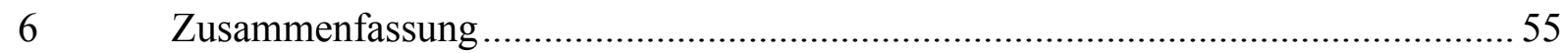

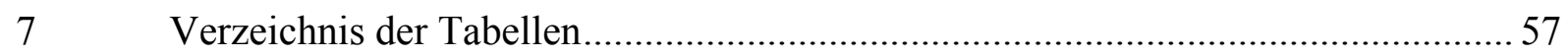

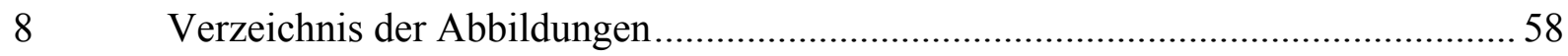


9

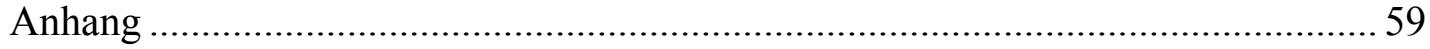

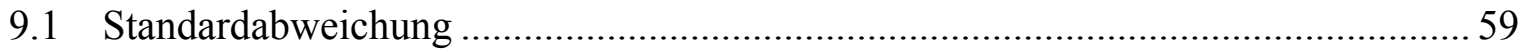

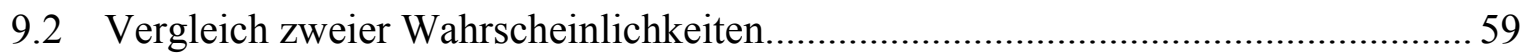

9.2.1 Wahrscheinlichkeit von Chromosomenanomalien im Falle des Vorliegens einer Pyelektasie, abhängig vom fetalen Geschlecht .................................... 59

9.2.2 Wahrscheinlichkeit von Chromosomenanomalien bei positivem Softmarkerbefund, abhängig vom mütterlichen Alter....................................59

9.2.3 Wahrscheinlichkeit einer Trisomie 18 bei Vorliegen von isolierten CPC (verglichen mit einem Kollektiv von GRAY et al. (1996)) 60

9.2.4 Unterschiede in der Wahrscheinlichkeit von Chromosomenanomalien in den

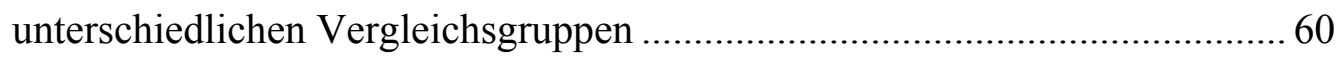

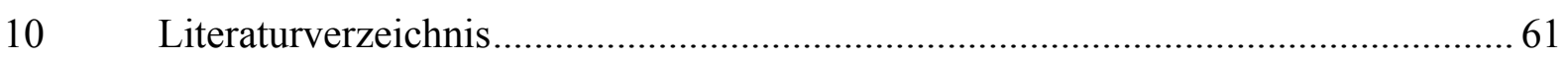




\section{Abkürzungsverzeichnis}

A.

arteria

Aa.

arteriae

$\mathrm{AC}$

amniocentesis $=$ Amniozentese

AFP

$\alpha$-Fetoprotein

a. p. anterior-posterior (Strahlengang)

BPD

biparietaler Durchmesser

BWS

Brustwirbelsäule

$\mathrm{CF}$

cystic fibrosis $=$ Zystische Fibrose (Mukoviszidose)

CMV

Zytomegalievirus

CPC

choroid plexus cysts $=$ Plexus-choroideus-Zysten

CVS

chorionic villus sampling $=$ Chorionzottenbiopsie

DNA

deoxyribonucleic acid $=$ Desoxyribonukleinsäure

FISH

Fluoreszenz-in-situ-Hybridisierung

GÖ

\section{Göttingen}

hCG

humanes Choriongonadotropin

ICEF

intrakardialer echogener Fokus

LKG

Lippen-Kiefer-Gaumen-Spalte

$\mathrm{MHz}$

Megahertz

$\mathrm{NOM}$

Northeim

NT

Nackentransparenz

PAPP-A

pregnancy-associated protein A

SD

standard deviation $=$ Standardabweichung

SUA

single umbilical artery $=$ singuläre Nabelschnurarterie

SSL

Scheitel-Steiß-Länge

SSW

Schwangerschaftswoche

$\mathrm{uE}_{3}$

unconjugated estriol $=$ freies Östriol

V.

vena

ZNS

Zentrales Nervensystem 


\section{Einleitung}

\subsection{Methoden und Indikationen der Pränataldiagnostik}

Unter dem Begriff der Pränataldiagnostik werden verschiedene invasive und nicht-invasive diagnostische Methoden und Screening-Verfahren zusammengefasst, welche der Überwachung des Schwangerschaftsverlaufs sowie der Früherkennung von fetalen Fehlbildungen dienen.

$\mathrm{Zu}$ den nicht-invasiven Methoden gehören:

- das (humangenetische) Beratungsgespräch

- die Bestimmung von Serumparametern bei der Schwangeren (PAPP-A-Test u. a.)

- die pränatale Risikoanalyse (siehe Kapitel 1.1.2.3, S. 3)

- der pränatale Ultraschall (siehe Kapitel 1.2, S. 6).

$\mathrm{Zu}$ den invasiven Methoden zählt man:

- $\quad$ die Amniozentese (amniocentesis $=\mathrm{AC})$

- die Chorionzottenbiopsie (chorionic villus sampling $=$ CVS)

- die Chordozentese (Nabelschnurpunktion zur Gewinnung von fetalem Blut).

Die Kombination aus einer Blutentnahme bei der Schwangeren und einer pränatalen Ultraschalluntersuchung wird auch als ,pränatale Risikoanalyse“ bezeichnet.

\subsubsection{Indikationen}

Die allgemeine Fehlbildungsrate $-\mathrm{d}$. h. das Risiko aller Schwangeren, ein Kind mit einer Fehlbildung zu bekommen - beträgt durchschnittlich 4 bis $5 \%$ (FELBERBAUM et al. 2005). Dabei haben schwere Fehlbildungen einen Anteil von ca. $1 \%$.

Zusätzlich gilt, dass das Fehlbildungsrisiko mit dem mütterlichen Alter steigt. So beträgt das Risiko einer 25jährigen Frau, ein Kind mit einer Trisomie 21 (Down-Syndrom) zu bekommen, etwa 1:1300 - dasjenige einer 35 Jahre alten Schwangeren liegt dahingegen bereits bei 1:380 (Hormon Zentrum München 2009).

Somit ist der betreuende Gynäkologe nach den Mutterschaftsrichtlinien dazu verpflichtet, jede Schwangere, die zum Zeitpunkt des errechneten Geburtstermins das 35. Lebensjahr erreicht hat, hinsichtlich ihres erhöhten Risikos zu beraten, und ihr eine vorgeburtliche Diagnostik anzubieten (,Altersindikation“). 
Nicht-invasive Methoden wie der Ultraschall und die Bestimmung von Serummarkern stehen dabei im Vordergrund. Mit zunehmendem mütterlichem Alter gewinnen jedoch auch die invasiven diagnostischen Methoden wie z. B. die Amniozentese zunehmend an Bedeutung.

Die Grenze wurde für ein mütterliches Alter von 35 Jahren festgesetzt, da zu diesem Zeitpunkt das Risiko einer fetalen Chromosomenanomalie dem Fehlgeburtsrisiko aufgrund einer invasiven diagnostischen Maßnahme entspricht.

Als Hauptindikationen für die invasive pränatale Diagnostik gelten im Allgemeinen:

- das erhöhte Alter der Schwangeren (> 35 Jahre)

- pathologische Serummarker (s. u.)

- ein auffälliger Ultraschallbefund

- $\quad$ eine vorausgegangene Geburt eines Kindes mit einer Chromosomenanomalie

- familiäre Stoffwechseldefekte oder andere erbliche Erkrankungen.

\subsubsection{Nicht-invasive Methoden}

\subsubsection{Das humangenetische Beratungsgespräch}

Ein humangenetisches Beratungsgespräch kann grundsätzlich von allen werdenden Eltern sowie von Paaren mit Kinderwunsch in Anspruch genommen werden.

Es dient der Information über die verschiedenen pränatalen Untersuchungsmöglichkeiten sowie der Beratung hinsichtlich erblicher Erkrankungen und deren Wiederholungsrisiko für die Nachkommen in einer betroffenen Familie.

Eine humangenetische Beratung erfolgt in der Regel durch qualifiziertes Fachpersonal wie z. B. Fachärzte für Humangenetik, Gynäkologie oder Pädiatrie in einer auf Pränatalmedizin und Humangenetik entsprechend spezialisierten Institution.

\subsubsection{Triple-Test und PAPP-A}

Beim so genannten „Triple-Test“, der üblicherweise in der 16. oder 17. Schwangerschaftswoche (SSW) durchgeführt wird, werden drei Marker im Serum der Schwangeren bestimmt:

- das humane Choriongonadotropin (hCG)

- das $\alpha$-Fetoprotein (AFP)

- $\quad$ das freie Östriol (unconjugated estriol $=\mathrm{uE}_{3}$ ).

Typisch für eine Trisomie 21 ist dabei z. B. eine deutliche Erhöhung des hCG bei gleichzeitig erniedrigten Werten für $\mathrm{AFP}$ und $\mathrm{uE}_{3}$. 
Das im Triple-Test enthaltene AFP dient zusätzlich als biochemischer Marker für fetale Verschlussstörungen (z. B. einen Neuralrohrdefekt).

Die kombinierte Bewertung des Triple-Tests zusammen mit dem mütterlichen Altersrisiko dient dazu, das individuelle Risiko einer Schwangeren für ein Kind mit einer Chromosomenanomalie (v. a. Trisomie 21 (Down-Syndrom) und Trisomie 18 (EDWARDSSyndrom)) zu präzisieren (SANCKEN 2000).

Der Triple-Test war das erste, routinemäßig in der Schwangerenbetreuung eingesetzte Screening-Instrument. Er wird heutzutage jedoch überwiegend von neueren Methoden der Risikopräzisierung abgelöst (siehe Kapitel 1.1.2.3, S. 3).

Ein weiterer wichtiger biochemischer Marker, dessen Veränderung auf eine fetale Chromosomenanomalie hinweisen kann, ist das pregnancy-associated plasma protein A (PAPP-A). Dieses sollte vorzugsweise zwischen der 10. und der 13. SSW bestimmt werden. Bei der Bestimmung des PAPP-A spricht eine Erniedrigung des Wertes für eine Trisomie 21.

\subsubsection{Pränatale Risikoanalyse}

Eine wesentliche Verbesserung der Möglichkeiten zur Risikopräzisierung wird durch die Kombination unterschiedlicher Methoden - beispielsweise die Kombination aus Blutuntersuchungen und pränatalem Ultraschall - erreicht.

Es werden folgende Untersuchungsverfahren unterschieden:

- das Ersttrimester-Screening

- das integrierte Screening

- das Quadruple-Screening.

Das Ersttrimester-Screening setzt sich aus der sonographischen Messung der fetalen Nackentransparenz (NT) und der Bestimmung von PAPP-A und freiem $\beta$-hCG im Serum der Schwangeren zusammen.

Sowohl die Ultraschall-Untersuchung als auch die Blutanalyse werden im I. Trimenon zwischen der 11. und der 14. SSW - vorgenommen.

Hierbei steigen z. B. die Detektionsraten für die häufigsten Chromosomenanomalien auf 80 \% (EiBeN und Glaubitz 2005, NiCOlaides 2005). In einer großen Studie mit 38167 untersuchten Schwangeren betrugen die Detektionsraten für ein Kind mit einer Trisomie 21 sogar $87 \%$ (11. SSW), $85 \%$ (12. SSW) und $82 \%$ (13. SSW) (MALONE et al. 2005).

Das so genannte ,integrierte Screening“ beinhaltet Untersuchungen, die sowohl im I. als auch im II. Trimenon durchgeführt werden. 
Im I. Trimenon (in der 10. oder 11. SSW) werden die fetale NT sowie das PAPP-A im maternalen Serum gemessen.

Im II. Trimenon (zwischen den SSW 14+0 und 17+6) erfolgt dann die Bestimmung von vier weiteren Blutwerten bei der Schwangeren: hCG, AFP, $\mathrm{uE}_{3}$ und Inhibin A.

Durch die Bestimmung dieser zwei zusätzlichen Blutwerte erhöht sich die Detektionsrate für ein Kind mit einem Down-Syndrom auf 96 \% (MALONE et al. 2005). Außerdem können durch die Bestimmung des AFP auch Feten mit einem Neuralrohrdefekt entdeckt werden.

Damit entspricht das integrierte Screening der Kombination aus der Messung der fetalen NT und dem Quadruple-Screening (s. u.).

Das Quadruple-Screening ist eine reine Blutuntersuchung der Schwangeren, welche im

II. Trimenon (zwischen der 14. und der 18. SSW) vorgenommen wird. Hierbei werden folgende Werte bestimmt: hCG, $\mathrm{AFP}, \mathrm{uE}_{3}$ und Inhibin A (s. o.).

Aus den Werten dieser Untersuchung und unter Berücksichtigung des mütterlichen Alters kann das individuelle Risiko für ein Kind mit einer Chromosomenanomalie berechnet werden. Die Detektionsrate für ein Kind mit einer Trisomie 21 wird hierbei mit $81 \%$ beziffert (MALONE et al. 2005).

\subsubsection{Invasive Methoden}

\subsubsection{Amniozentese, Chorionzottenbiopsie und Chordozentese}

Eine Amniozentese wird meist in der 16. oder 17. Schwangerschaftswoche (SSW) durchgeführt.

Sie dient der Gewinnung von fetalen Zellen aus dem Fruchtwasser, welches mittels sonographisch gesteuerter Punktion der Fruchthöhle durch die mütterliche Bauchdecke gewonnen wird. Bei den Amnionzellen handelt es sich um fetale Zellen der Dermis sowie des Magen-Darm- und des Urogenitaltraktes.

Aus den Zellkernen der auf diese Weise gewonnenen Zellen können die fetalen Chromosomen dann isoliert und schließlich analysiert werden. Mit dem Ergebnis der Chromosomenanalyse ist hierbei ca. 12 Tage nach der Punktion zu rechnen.

Eine Möglichkeit, das Ergebnis der Chromosomenanalyse früher zu erhalten, bietet die Fluoreszenz-in-situ-Hybridisierung (FISH).

Diese Methode dient zwar als Schnelltest zur ersten Orientierung, mit ihrer Hilfe können aber lediglich numerische Chromosomenaberrationen erfasst werden. 
Mittels FISH werden die Chromosomen 13, 18, 21, X und $\mathrm{Y}$ untersucht, da diese am häufigsten von Anomalien betroffen sind. Außerdem steigt das Risiko für das Vorliegen fetaler numerischer Aberrationen eben dieser Chromosomen mit zunehmendem mütterlichem Alter an.

Auch die Chorionzottenbiopsie (ab der 10. SSW), bei der ein kleines Stück des Plazentagewebes entnommen wird, wie auch die Entnahme von Fetalblut aus der Nabelschnur (in der 17. oder 18. SSW) haben die fetale Chromosomenanalyse zum Ziel.

Ebenso wie die Amniozentese werden auch diese beiden Untersuchungen unter sonographischer Kontrolle durchgeführt. Das Ergebnis der Chromosomenanalyse nach einer Chorionzottenbiopsie liegt bereits wenige Tage nach dem Eingriff vor.

Die Chordozentese wird meist nicht primär zur Gewinnung fetaler Zellen mit dem Ziel der Chromosomenanalyse durchgeführt. Sie dient in den späteren Schwangerschaftswochen vielmehr der Erkennung einer fetalen Anämie, einer Rhesusinkompatibilität oder einer kindlichen Infektion.

Bei Vorliegen z. B. einer fetalen Anämie ist es außerdem möglich, mittels Chordozentese eine Bluttransfusion zur intrauterinen fetalen Therapie durchzuführen.

Mithilfe der invasiven Diagnostik kann - bei Vorliegen einer erblichen Erkrankung in den Familien der werdenden Eltern - die DNA aus den entnommenen fetalen Zellen außerdem gezielt auf den entsprechenden Gendefekt hin untersucht werden.

Anders als bei den nicht-invasiven Methoden, welche lediglich Screening-Untersuchungen darstellen, handelt es sich bei den hier beschriebenen invasiven Methoden um diagnostische Verfahren.

Das bedeutet, diese Untersuchungen geben definitiv Auskunft darüber, ob der Fet Träger einer Chromosomenanomalie ist, oder nicht.

\subsubsection{Risiken der invasiven Pränataldiagnosik}

Die Hauptrisiken der invasiven pränataldiagnostischen Methoden bestehen in der Induktion einer Fehlgeburt oder eines vorzeitigen Blasensprungs.

Sie sind abhängig von der Art, von der Punktionsweise (transvaginal / -zervikal oder transabdominal) und vom Zeitpunkt des durchgeführten Eingriffs.

Auch durch den Eingriff verursachte Infektionen sind nicht zu unterschätzen, da sie ebenfalls eine Gefahr für Mutter und Kind darstellen, und eine Fehlgeburt verursachen können. 
Die Rate schwerer Komplikationen, die zum Abort oder zum Tod in utero führen, beträgt innerhalb von 14 Tagen nach Amniozentese 0,74 \%, nach Chorionzottenbiopsie $0,99 \%$ und nach Chordozentese 0,75\% (BETTELHEIM et al. 2002).

Ähnliche Komplikationsraten beschreiben auch MuJEZINOVIC und AlfIREVIC (2007), die nach der Evaluation von insgesamt 45 Studien Fehlgeburtsraten von 0,6 \% nach Amniozentese und 0,7 \% nach Chorionzottenbiopsie errechnet haben.

\subsection{Die Bedeutung des pränatalen Ultraschalls}

Die Sonographie gehört seit vielen Jahren zu den Routineuntersuchungen während der Schwangerschaft.

Sie ist unerlässlich als diagnostisches Instrument in der Pränataldiagnostik, da bisher - im Gegensatz z. B. zu Röntgenstrahlen - kein Schädigungspotenzial für den Feten nachgewiesen werden konnte.

Somit ist die Sonographie momentan das einzige bildgebende Verfahren, das in der Schwangerschaft ohne Bedenken angewandt werden kann. Laut European Committee for Ultrasonic Radiation Safety sollte die sonographische Darstellung von Embryonen dennoch auf die notwendige Untersuchungszeit beschränkt werden (DIRKS 2004).

Als nicht-invasive und damit komplikationsarme Methode wird der Ultraschall von den Patientinnen zudem gut toleriert.

Darüber hinaus ist er leicht zugänglich und relativ kostengünstig, was seinen Einsatz als Standarduntersuchung in der Schwangerschaftsvorsorge natürlich weiterhin begünstigt.

In den Richtlinien für die Mutterschaftsvorsorge sind drei Ultraschalluntersuchungen vorgesehen: eine in der Frühschwangerschaft (in der 9. bis 12. SSW), eine im II. Trimenon (zwischen der 19. und der 22. SSW) und eine im III. Trimenon (in der 29. bis 32. SSW) (Gemeinsamer Bundesausschuss 2009).

Im I. Trimenon erfolgt die sonographische Untersuchung in aller Regel transvaginal, da in diesem frühen Schwangerschaftsstadium so die höchste Detailgenauigkeit erreicht werden kann.

Ziel der Untersuchung ist die Feststellung des Schwangerschaftsalters, der Nachweis bzw. Ausschluss der intrakavitären Lage des Embryos sowie der Nachweis einer evtl. vorliegenden Mehrlingsschwangerschaft. 
Die im II. Trimenon vorgesehene Ultraschalluntersuchung dient der Beurteilung des fetalen Wachstums, der Fruchtwassermenge, der Plazentalokalisation und -struktur sowie der Feststellung grober Fehlbildungen.

Finden sich bei dieser Untersuchung Hinweise auf eine fetale Fehlbildung oder einen gestörten Schwangerschaftsverlauf (z. B. Abweichungen in der Fruchtwassermenge oder der fetalen Weiterentwicklung), erfolgt die Überweisung der Schwangeren an ein pränataldiagnostisches Zentrum zum detaillierten Fehlbildungsausschluss (sog. „Feindiagnostik“, siehe Kapitel 1.3, S. 7).

Ziel der letzten sonographischen Untersuchung im III. Trimenon ist der Nachweis einer zeitgerechten Organ- und Größenentwicklung des Ungeborenen sowie die Bestimmung der Plazentalokalisation, der Fruchtwassermenge und der Lage des Feten.

Hierdurch können potentielle Geburtskomplikationen erfasst und entsprechende Vorbereitungen getroffen werden.

\subsection{Die sonographische Feindiagnostik}

Die sonographische Feindiagnostik - auch „Feinultraschall“, „,sonographischer Fehlbildungsultraschall“ oder „Organscreening“ genannt - wird in der Regel zwischen der 19. und der 22. Schwangerschaftswoche (SSW) vorgenommen.

Sie wird bevorzugt in diesem Zeitraum durchgeführt, da hier das Verhältnis zwischen der Größe der fetalen Strukturen, d. h. der Detailgenauigkeit, und der Übersichtlichkeit am günstigsten ist (FRIESE o. J.).

Die Untersuchung sollte die Durchmusterung aller fetalen Organsysteme sowie die Beurteilung ihrer Funktion beinhalten.

Ergänzt werden kann dieses transabdominale Sonogramm durch die farbcodierte Dopplersonographie, welche eine noch genauere Diagnostik bestimmter fetaler Funktionssysteme erlaubt. 
Die im Rahmen der sonographischen Feindiagnostik untersuchten Strukturen zeigt Tab. 1:

\begin{tabular}{|l|l|}
\hline Untersuchte Region & Besonderheiten \\
\hline Kopf & $\begin{array}{l}\text { Zentrales Nervensystem (ZNS) } \\
\text { Schädelform }\end{array}$ \\
\hline Skelett & Wirbelsäule \\
& Extremitäten \\
\hline Thorax & Herz \\
& Lungen \\
\hline Abdomen & Gastrointestinaltrakt \\
& Bauchwand \\
\hline Urogenitaltrakt & Nieren \\
& ableitende Harnwege \\
& äußeres Genitale \\
\hline Nabelschnur & Aa. umbilicales \\
& V. umbilicalis \\
\hline Uterus & Plazenta \\
& Aa. uterinae \\
\hline
\end{tabular}

Tab. 1: Organsysteme, die im Rahmen der sonographischen Feindiagnostik untersucht werden

Diese detaillierte Untersuchung erlaubt es dem hierfür qualifizierten und erfahrenen Untersucher, Entwicklungsstörungen einzelner Organsysteme sowie anatomische Besonderheiten beim Feten festzustellen bzw. auszuschließen.

Außerdem können so genannte „,Sonographische Softmarker“ (siehe Kapitel 1.4, S. 9) erkannt und näher bestimmt bzw. ausgeschlossen werden.

Auch, wenn Chromosomenaberrationen im pränatalen Ultraschall natürlich nicht definitiv nachgewiesen werden können, so kann doch die Kombination mehrerer Auffälligkeiten den Verdacht auf eine solche Anomalie erhärten.

In einem solchen Fall muss mit der Patientin sorgfältig diskutiert werden, ob weitere invasive diagnostische Verfahren angewandt werden sollten, um Gewissheit über den Gesundheitszustand des Feten zu erhalten. 


\subsection{Sonographische Softmarker im pränatalen Ultraschall}

Wie oben bereits beschrieben, ist ein Ziel der Pränataldiagnostik, das Vorliegen von Chromosomenanomalien und anderen Erkrankungen wie z. B. Herzfehler, Omphalozele, Gastroschisis, Neuralrohrdefekte u. a. beim Feten frühzeitig zu entdecken.

Das Wissen um eine (evtl. schwerwiegende) Krankheit des Ungeborenen gibt sowohl den werdenden Eltern als auch den betreuenden Ärzten die Möglichkeit, das weitere Vorgehen in Ruhe miteinander zu diskutieren.

So kann z. B. geplant werden, die Geburt in einer spezialisierten Klinik mit einer ausreichenden pädiatrischen Versorgung durchzuführen. Außerdem wird den werdenden Eltern so mehr Zeit gegeben, sich auf das Leben mit einem kranken Kind vorzubereiten.

Da die invasiven diagnostischen Methoden mit einem (wenn auch relativ geringen) Abortrisiko behaftet sind (s. o.), besteht allgemein natürlich der Wunsch nach einem nicht-invasiven Untersuchungsverfahren, welches es ebenfalls ermöglicht, fetale Chromosomenanomalien rechtzeitig zu erkennen.

Der bereits erwähnte Triple-Test beispielsweise ist zwar ein solches nicht-invasives Verfahren, das - in Zusammenschau mit anderen Risikofaktoren wie z. B. dem erhöhten mütterlichen Alter - eine gewisse Risikokalkulation für das Vorliegen einer Chromosomenaberration erlaubt. Da der Triple-Test aber lediglich eine Methode zur Risikopräzisierung darstellt, kann er eine weiterführende (ggf. auch invasive) Diagnostik nicht ersetzen.

So ruht die Hoffnung also auf der pränatalen Sonographie, vor allem, da sich die Medizintechnologie ständig weiterentwickelt und die Ultraschallgeräte somit immer bessere und hochauflösendere Bilder liefern.

Aufgrund dieser verbesserten Qualität ist es seit einigen Jahren möglich, neben den „Hardmarkern“ (Fehlbildungen wie z. B. Herzfehler, Duodenalatresie, Zwerchfelldefekte) so genannte „Softmarker“ zu diagnostizieren.

Als sonographische Softmarker bezeichnet man geringgradige Strukturauffälligkeiten in der Anatomie des Feten, welche nicht spezifisch sind und häufig nur vorübergehend auftreten (RANIGA et al. 2006).

Sie können am besten zwischen der 16. und der 20. SSW diagnostiziert werden. Außerdem ist das Auftreten von Softmarkern in einem gewissen Prozentsatz mit dem Vorliegen von Chromosomenanomalien assoziiert. 
Tab. 2 zeigt Beispiele für gängige Softmarker:

\begin{tabular}{|c|c|}
\hline Organsystem & Softmarker \\
\hline Zentrales Nervensystem & $\begin{array}{l}\text { Plexus-choroideus-Zysten (CPC) } \\
\text { milde Ventrikulomegalie } \\
\text { erweiterte Cisterna magna } \\
\text { offener Vermis cerebelli }\end{array}$ \\
\hline Kopf / Hals & $\begin{array}{l}\text { erhöhte Nackentransparenz (NT) } \\
\text { unübliche Kopfform / -größe } \\
\text { hypoplastisches Nasenbein }\end{array}$ \\
\hline Herz & white spots („,Golfballphänomen“) \\
\hline Gastrointestinaltrakt & $\begin{array}{l}\text { hyperechogener Darm } \\
\text { „double-bubble-Phänomen“ }\end{array}$ \\
\hline Urogenitaltrakt & $\begin{array}{l}\text { milde Pyelektasie } \\
\text { hyperechogene Nieren }\end{array}$ \\
\hline Extremitäten & $\begin{array}{l}\text { kurzes Femur / kurzer Humerus } \\
\text { clenched fist (geballte Faust) } \\
\text { Sandalenlücke / -furche }\end{array}$ \\
\hline Sonstiges & $\begin{array}{l}\text { singuläre Nabelschnurarterie (SUA) } \\
\text { Poly- / Oligohydramnion }\end{array}$ \\
\hline
\end{tabular}

Tab. 2: Beispiele für gängige Softmarker

Während isoliert auftretende Softmarker häufig als harmlos eingestuft werden, erhöht vor allem das gleichzeitige Vorliegen mehrerer Softmarker oder die Kombination mit zusätzlichen Anomalien das Risiko für eine fetale Aneuploidie dramatisch (BROMLEY et al. 2002).

Diese Tatsache macht sie zu einem wertvollen Screeningparameter in der Pränataldiagnostik. 


\subsection{Definition und Diagnostik sonographischer Softmarker}

\subsubsection{Pyelektasie}

Unter einer Pyelektasie versteht man ein hypoechogenes Areal im Bereich des Nierenbeckens des Feten, welches ein- oder auch beidseitig vorliegen kann.

Um eine Pyelektasie diagnostizieren zu können, wird das fetale Nierenbecken allgemein im anterior-posterior (a. p.)-Schnittbild ausgemessen.

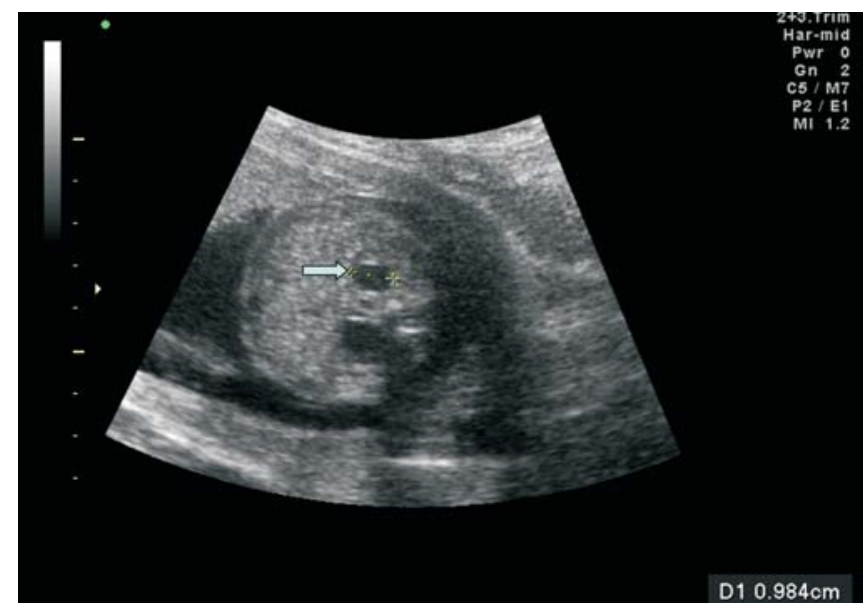

Abb. 1: Bilaterale renale Pyelektasie (a. p.) (VAN DEN HoF et al. 2005, S. 596)

Uneinigkeit besteht dahingegen bei der Frage, ab welchem Durchmesser man überhaupt von dem Vorliegen einer Pyelektasie spricht, und wann sie lediglich als „mild“ bezeichnet wird.

Sehr genau ist die Unterteilung in mild ( $>5$ bis $\leq 7 \mathrm{~mm}$ ), moderat $(>7$ bis $\leq 10 \mathrm{~mm}$ ) und schwer (>10 mm) in der 20. SSW (AHMAD und GREEN 2005).

Andere Autoren sprechen insgesamt von einer milden Pyelektasie bei einem a. p.-Durchmesser von 5 bis $10 \mathrm{~mm}$ (DAMEN-ELIAS et al. 2005) oder von $\geq 4$ bis $<$ bzw. $\leq 10 \mathrm{~mm}$ vor der 28. SSW (COHEN-OverbeEK et al. 2005, Persutte et al. 1997). 


\subsubsection{Plexus-choroideus-Zysten (CPC)}

Als Plexus-choroideus-Zysten (choroid plexus cysts $=$ CPC) bezeichnet man kleine Zysten $(\geq 3 \mathrm{~mm}$ ) im Bereich des Plexus choroideus in den fetalen Seitenventrikeln, welche im Transversalschnitt des fetalen Kopfes zwischen der 14. und der 24. SSW sonographisch diagnostiziert werden können (CHITTY et al. 1998).

In anderen Studien gelten erst Durchmesser von $>5 \mathrm{~mm}$ in der $\geq 19$. SSW als Einschlusskriterium für Plexuszysten (WALKINSHAW et al. 1994).

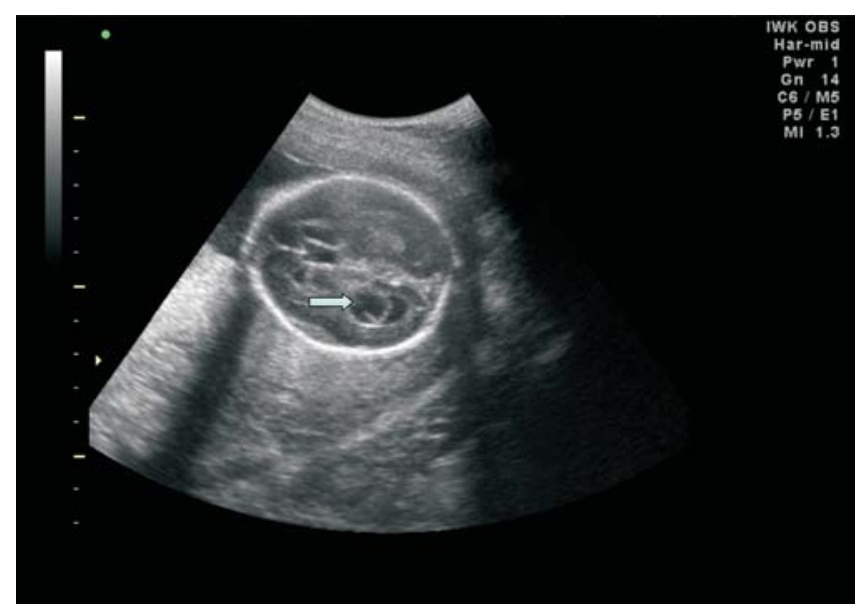

Abb. 2: Plexus-choroideus-Zyste (VAN DEN HoF et al. 2005, S. 604)

Es sollten stets beide Seitenventrikel auf das Vorhandensein von Zysten hin untersucht werden.

Dabei sind die Lateralität, die Position und die Anzahl der Zysten aber anscheinend nicht von klinischer Relevanz (GEARY et al. 1997, GRAY et al. 1996). Allerdings sehen GRAY et al. (1996) eine signifikante Assoziation zwischen der Größe der Zysten ( $\geq 10 \mathrm{~mm}$ ) und dem Auftreten einer Trisomie 18 (EDWARDS-Syndrom). 


\subsubsection{Ventrikulomegalie}

Eine zerebrale Ventrikulomegalie liegt definitionsgemäß ab einer Größe des Atriums des fetalen Seitenventrikels von $\geq 10 \mathrm{~mm}$ vor (SENAT et al. 1999, FiLly et al. 1989, CARDOZA et al. 1988).

Von einer milden (,borderline“) Ventrikulomegalie spricht man bei Durchmessern von 10 bis $15 \mathrm{~mm}$ (PILU et al. 1999) oder auch 11 bis $15 \mathrm{~mm}$ (TOMLINSON et al. 1997).

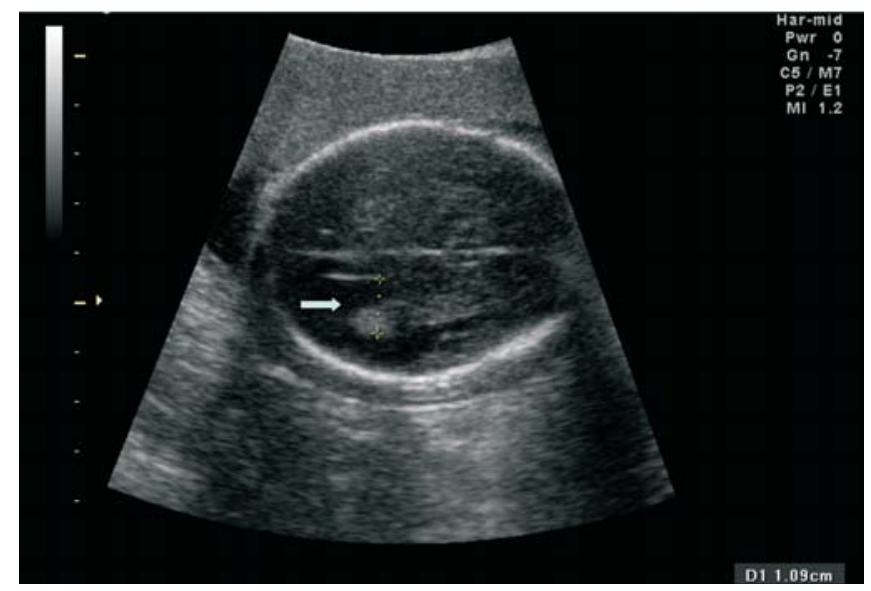

Abb. 3: Leicht erweitertes Hinterhorn des Seitenventrikels (VAN DEN HoF et al. 2005, S. 602)

Die Messung des atrialen Ventrikeldurchmessers sollte in der Transversalebene des fetalen Kopfes auf Höhe der Nuclei thalamici erfolgen (VAN DEN HOF et al. 2005).

\subsubsection{Kurzes Femur / kurzer Humerus}

Man spricht von einem kurzen Femur oder einem kurzen Humerus als sonographischem Softmarker, wenn die Femur- / Humeruslänge des Feten eines der beiden folgenden Kriterien erfüllt:

- entweder, die Femur- / Humeruslänge liegt unter der 5. Perzentile der für das Gestationsalter üblichen Länge (VAN DEN HOF et al. 2005)

- oder, das Femur / der Humerus misst weniger als die aufgrund des biparietalen Durchmessers zu erwartende Länge (TANNIRANDORN et al. 2002, NYBERG et al. 1993 b).

Beim Ausmessen der Femur- / Humeruslänge sollten die Epiphysen im Ultraschall sichtbar sein, aber nicht in die Messung mit einbezogen werden (VAN DEN HoF et al. 2005). 


\subsubsection{Intrakardialer echogener Fokus (ICEF)}

Als intrakardiale echogene Foci (intracardiac echogenic focus = ICEF) - auch „golf balls“ oder „white spots“ genannt - bezeichnet man sonographisch echoreiche Strukturen im Bereich der Papillarmuskeln des fetalen Herzens, welche mittels Einstellung des Vierkammerblicks diagnostiziert werden können.

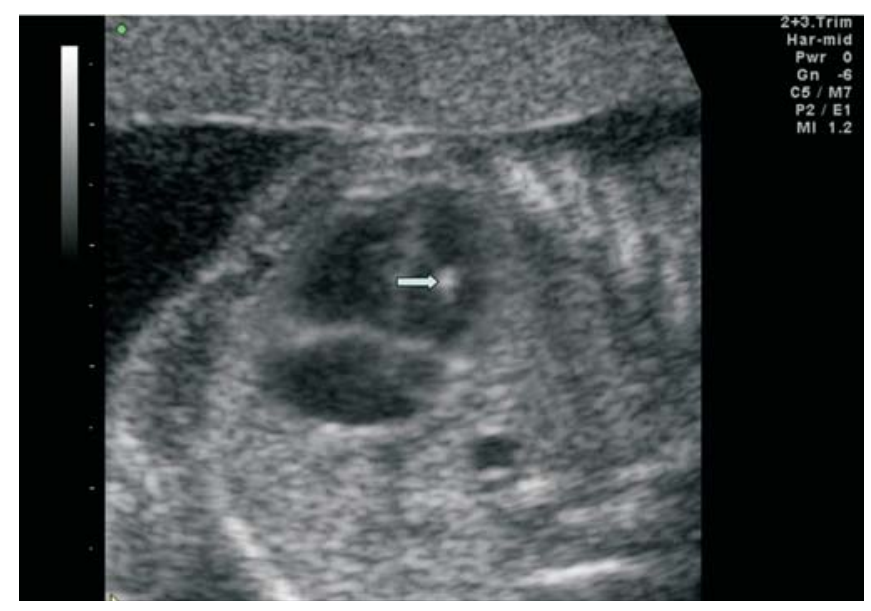

Abb. 4: Echogener intrakardialer Fokus im linken Ventrikel (VAN DEN HoF et al. 2005, S. 595)

Die Echogenität dieser Foci wird in Bezug zu derjenigen der umgebenden knöchernen Strukturen gesetzt, und anhand eines Schemas (WAX et al. 2000) in verschiedene Grade eingeteilt (siehe Tab. 3).

\begin{tabular}{|l|l|}
\hline Grad & Beschreibung \\
\hline 0 & keine ICEF vorhanden \\
\hline 1 & bei Reduktion der Verstärkung (gain) verschwinden die ICEF vor der BWS \\
\hline 2 & bei Reduktion der Verstärkung verschwinden die ICEF zum selben Zeitpunkt wie die BWS \\
\hline 3 & bei Reduktion der Verstärkung verschwinden die ICEF erst nach der BWS \\
\hline
\end{tabular}

Tab. 3: Gradeinteilung der ICEF (WAX et al. 2000) 
$78 \%$ der ICEF finden sich ausschließlich im linken und $18 \%$ im rechten Ventrikel. In $4 \%$ der Fälle findet man echogene Foci gleichzeitig in beiden Ventrikeln (WOLMAN et al. 2000). Ähnliche Angaben finden sich bei WAX et al. (2000) mit $83 \%$ der ICEF im linken und $17 \%$ im rechten Ventrikel.

\subsubsection{Singuläre Nabelschnurarterie (SUA)}

Die Nabelschnur enthält drei Gefäße: zwei Aa. umbilicales und eine V. umbilicalis. Bei Fehlen einer der zwei Arterien spricht man von einer singulären Nabelschnurarterie (single umbilical artery $=$ SUA).

Insgesamt fehlt die linke A. umbilicalis häufiger als die rechte: bei GEIPEL et al. (2000) fehlt die linke Arterie in 69,6 \% und die rechte in 30,4 \% der Fälle, bei ABUHAMAD et al. (1995) lauten die entsprechenden Angaben 75 \% für die linke und $27 \%$ für die rechte A. umbilicalis.

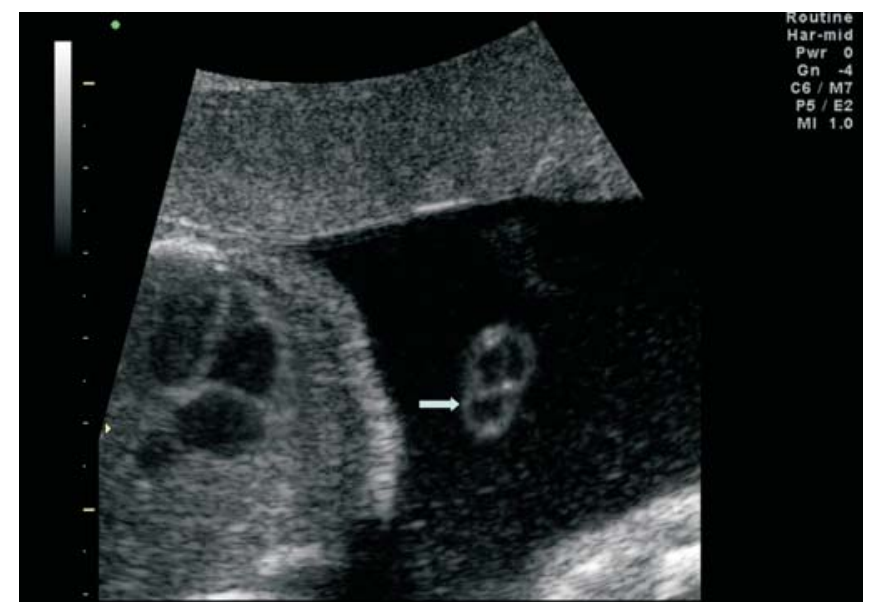

Abb. 5: SUA (im Querschnitt der Nabelschnur) (VAN DEN HoF et al. 2005, S. 598)

Die Nabelschnurgefäße können sonographisch sowohl im Längs-, als auch im Querschnitt beurteilt werden: entweder an der frei schwimmenden Nabelschnur, an ihrer Insertion am fetalen Abdomen oder an den Seiten der fetalen Harnblase.

Zur genaueren Diagnostik ist die Darstellung der Gefäße mittels Farbdoppler-Sonographie hilfreich. 


\subsubsection{Echogener Darm}

Vom so genannten „echogenen Darm“ spricht man bei Vorliegen von homogenen, hyperechogenen Arealen im fetalen Darm, welche entweder einzeln (fokal) oder multifokal auftreten können (AL-KOUATLY et al. 2001).

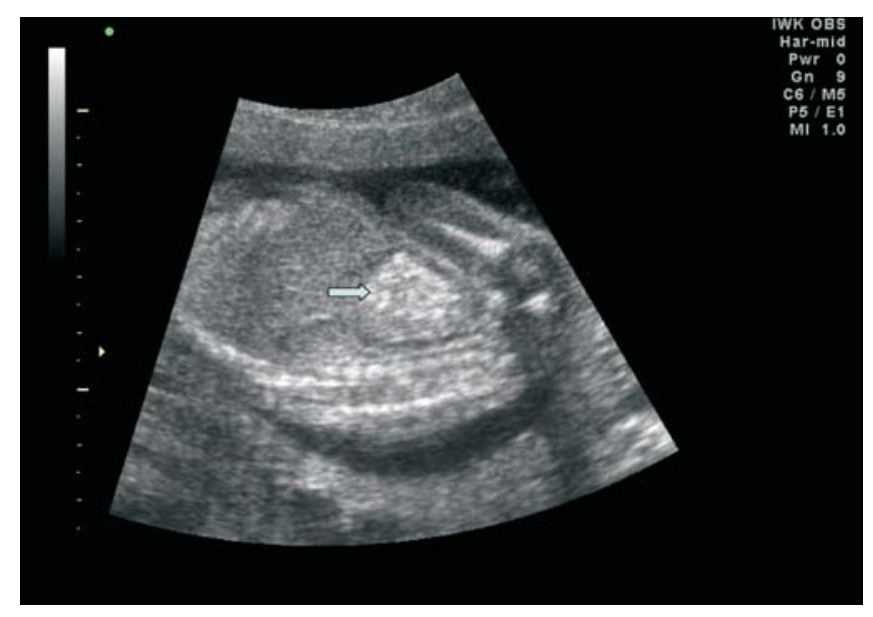

Abb. 6: Fetaler echogener Darm (VAN DEN HoF et al. 2005, S. 599)

Dabei spricht man von einem hyperechogenen Darm, wenn die Echogenität der Darmareale gleich oder größer als diejenige der umgebenden knöchernen Strukturen ist (GHOSE et al. 2000, DiCKE und CRANE 1992).

Einige Autoren vergleichen die Echogenität des Darms aber auch mit derjenigen der fetalen Leber (FonT und SolARi 1998, SEPUlVEDA et al. 1996, NYBERG et al. 1993 a).

Eine Gradeinteilung sollte möglichst immer angewandt werden, um den Befund dieses Softmarkers objektivieren zu können, und um Unterschiede in der Beurteilung durch verschiedene Untersucher zu minimieren.

\subsubsection{Sonderfall Nackenödem / erhöhte Nackentransparenz (NT)}

Als Nackenödem bezeichnet man eine Flüssigkeitsansammlung zwischen der Haut und dem darunter liegenden Bindegewebe im Bereich der fetalen Halswirbelsäule, welche im pränatalen Ultraschall diagnostiziert werden kann. Man spricht dann von dem Vorliegen einer „erhöhten Nackentransparenz (NT)““.

Die Nackentransparenz kann in den meisten Schwangerschaften im Verlauf des I. und frühen II. Trimenons gemessen werden (JACKSON und ROSE 1998). In diesem Zeitraum entwickelt sich das fetale Lymphsystem; die Nieren des Ungeborenen können die entstehende 
Lymphflüssigkeit allerdings noch nicht abtransportieren, sodass diese sich unter der sehr elastischen Nackenhaut ansammelt.

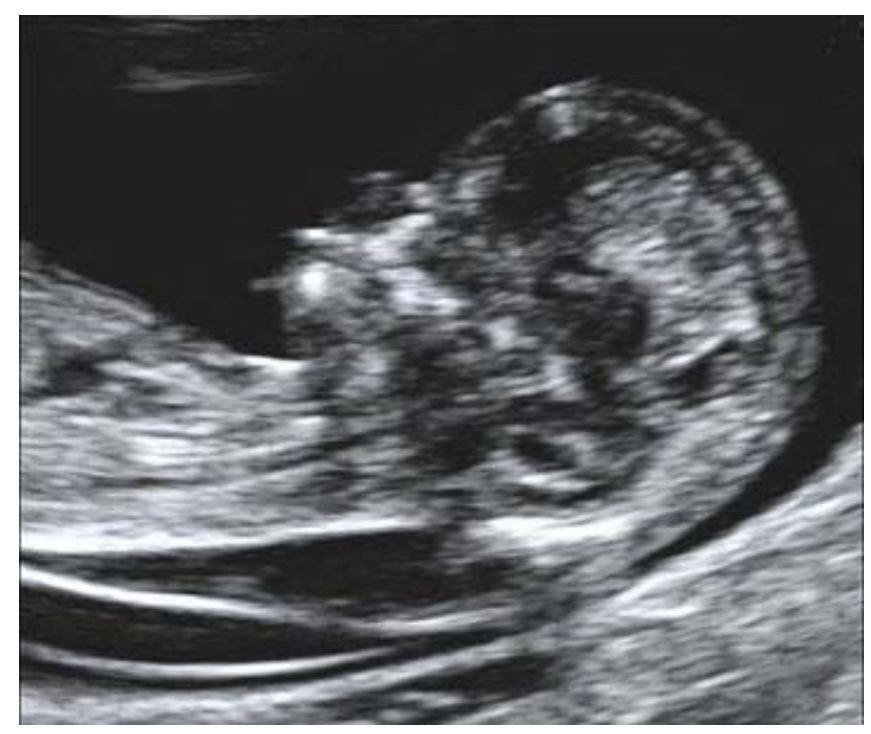

Abb. 7: Erhöhte Nackentransparenz (The Fetal Medicine Foundation 2009)

Die NT wird im medianen Sagittalschnitt bei einer Scheitel-Steiß-Länge (SSL) von 45-84 mm und einer korrekten Positionierung der Messpunkte (innen - innen) bestimmt (TERCANLI et al. 2002). Als auffällig wird die Nackentransparenz erst ab einem sagittalen Durchmesser von $\geq 2,5 \mathrm{~mm}$ (JEMMALI et al. 1999) oder auch $\geq 3 \mathrm{~mm}$ angesehen (BORRUTO et al. 2002, FELICETTI et al. 2001, NiCOLAIDES et al. 1992). 


\subsection{Sonographische Softmarker und fetale Chromosomenanomalien}

Seit der Erkenntnis, dass das Auftreten bestimmter Softmarker mit dem Vorliegen fetaler Chromosomenanomalien assoziiert sein kann, wird dieses Thema unter Gynäkologen, Pädiatern und Humangenetikern heftig diskutiert.

Dabei gehen die Meinungen der Wissenschaftler zum Teil sehr weit auseinander: während einige Autoren bestimmte Softmarker lediglich als strukturelle Normvarianten deuten, empfehlen andere dahingegen in jedem Fall eine weitergehende Diagnostik zum Ausschluss einer Chromosomenaberration.

Auch die Angaben zur Prävalenz der einzelnen Softmarker sowie zu der Wahrscheinlichkeit einer Assoziation zwischen ihnen und einer fetalen Chromosomenanomalie variieren sehr stark.

Diese Uneinigkeit führt nicht nur bei den werdenden Eltern, sondern auch bei den sie betreuenden Pränatalmedizinern zu großer Unsicherheit.

Es bestehen bis jetzt keine einheitlichen oder verbindlichen Empfehlungen, ob im Falle des Vorliegens eines oder mehrerer Softmarker im pränatalen Ultraschall weitere, invasive Methoden zum Fehlbildungsausschluss durchgeführt werden sollten. 


\section{Aufgabenstellung und Zielsetzung}

In der Vergangenheit sind zahlreiche Studien in dem Bestreben durchgeführt worden, den in der Pränatalmedizin tätigen Ärzten, Genetikern und Psychologen einen Leitfaden für das weitere Vorgehen im Falle der Entdeckung sonographischer Softmarker an die Hand zu geben.

Aufgrund der stark variierenden Angaben über die Assoziationsraten zwischen Softmarkern und fetalen Chromosomenanomalien konnte dieses Vorhaben bisher aber noch nicht zufrieden stellend erfüllt werden.

In der vorliegenden Arbeit sollen deshalb folgende Fragen untersucht werden:

- Wie hoch ist die Wahrscheinlichkeit für das Vorliegen einer Chromosomenaberration beim Feten, wenn ein bestimmter Softmarker entdeckt wird?

- Zu welchem Ergebnis führt die Amniozentese?

- Sollten grundsätzlich weitere, invasive diagnostische Methoden zum Fehlbildungsausschluss empfohlen werden, wenn im pränatalen Ultraschall ein bestimmter Softmarker entdeckt wird?

- Falls dem so ist: bei welchen Softmarkern sollte dies empfohlen werden? 


\section{Patientinnen und Methode}

\subsection{Patientinnen}

Im Zeitraum von 1993 bis 2003 wurden die an zwei pränatalmedizinischen Zentren durchgeführten Amniozentesen $\geq 15$. Schwangerschaftswoche (SSW) retrospektiv evaluiert. Die beiden teilnehmenden Zentren waren:

- die Klinik für Gynäkologie und Geburtshilfe der Universität Göttingen

- die Frauenklinik des Albert-Schweitzer-Krankenhauses Northeim.

Für die vorliegende Arbeit wurden insgesamt 683 der durchgeführten Amniozentesen ausgewertet. Die in die Studie eingeschlossenen Schwangeren wurden in zwei Gruppen aufgeteilt:

In der ersten, 348 Patientinnen (51,0 \%) umfassenden Gruppe, wurde die Amniozentese ausschließlich wegen eines oder mehrerer sonographischer Softmarker vorgenommen. Somit wiesen alle Feten, die in diese Gruppe eingeteilt wurden, keinerlei zusätzliche sonographische Auffälligkeiten auf.

Bei den übrigen 335 Patientinnen (49,0 \%) erfolgte die Amniozentese wegen fetaler Fehlbildungen oder anderer Marker (siehe Kapitel 3.1.2, S. 21). Dieses Kriterium der Gruppeneinteilung hatte zur Folge, dass einige der in diese Gruppe eingeteilten Feten zusätzlich auch sonographische Softmarker aufwiesen. In diesem Fall wurden die Softmarker jedoch als vernachlässigbar angesehen, da die echten fetalen Fehlbildungen („Hardmarker“) bereits den entscheidenden Ausschlag für die Indikationsstellung zur Durchführung einer Amniozentese gaben.

Abzüglich der 89 Feten mit einer erhöhten Nackentransparenz (siehe Kapitel 3.1.1, S. 20) konnten wir für diese Arbeit schließlich auf die Sonographieprotokolle von insgesamt 594 Schwangeren zurückgreifen.

\subsubsection{Sonderfall erhöhte Nackentransparenz (NT)}

Eine Nackentransparenzmessung gehört in Deutschland nicht zu den üblichen Vorsorgeuntersuchungen während der Schwangerschaft.

Die Messung der Nackentransparenz ist ein wesentlicher Bestandteil des ErsttrimesterScreenings, welches zusätzlich die Bestimmung von PAPP-A und freiem $\beta$-HCG im Serum der Schwangeren beinhaltet (siehe Kapitel 1.1.2.3, S. 3). In diesem Rahmen fließt die sonographisch ermittelte Nackentransparenz in die pränatale Risikoanalyse ein. 
Sowohl die Ultraschall-Untersuchung als auch die Blutanalyse werden im I. Trimenon zwischen der 11. und der 14. SSW - vorgenommen. Hierbei steigen z. B. die Detektionsraten für die häufigsten Chromosomenanomalien auf $80 \%$ (EIBEN und GLAUBITZ 2005, NiCOLAIDES 2005).

Durch die Bestimmung der NT sowie der Serumparameter des I. in Kombination mit Serumparametern des II. Trimenons (,,integriertes Screening“) erhöht sich die Detektionsrate für ein Kind mit einem Down-Syndrom auf $96 \%$ (MALONE et al. 2005).

Bei der erhöhten Nackentransparenz handelt es sich somit um einen wichtigen sonographischen Softmarker v. a. des I. Trimesters. Je breiter die NT ist, desto höher wird das individuelle Risiko für eine Chromosomenaberration nach einem für diese Risikoanalyse hinterlegten Algorithmus (hier werden noch die gemessenen Konzentrationen von PAPP-A und $\beta$-HCG, Alter, Gewicht u. v. m. berücksichtigt) berechnet, und umgekehrt.

Die NT wird nur noch selten isoliert betrachtet, sondern, wie oben ausgeführt, vielmehr im Rahmen der pränatalen Risikoanalyse mitbeurteilt, weshalb wir die NT bewusst aus der weiteren Beurteilung für die vorliegende Arbeit herausgenommen haben.

\subsubsection{Vergleichskollektiv der Feten mit echten Fehlbildungen und anderen Markern}

Softmarker sind sonographische Merkmale, die mit einer statistisch gesehen leichten Erhöhung der Wahrscheinlichkeit für eine Chromosomenaberration und / oder körperliche Fehlbildungen und / oder bestimmte Erkrankungen beim Feten in Verbindung gebracht werden. Sie kommen häufiger bei Feten mit einer Trisomie 21 vor und erhöhen so das relative Risiko für ein Down-Syndrom.

Für die vorliegende Arbeit wurden neben den Daten der Feten mit sonographischen Softmarkern auch Ultraschallbefunde von Feten mit schweren Fehlbildungen (sog. „Hardmarker") ausgewertet. Dabei wurden die Häufigkeit und das Spektrum der diagnostizierten Chromosomenaberrationen bei Feten mit sonographischen Hardmarkern mit denjenigen der Feten mit Softmarkern verglichen. 


\subsection{Methode}

Für die vorliegende Arbeit wurden die folgenden sonographischen Softmarker, fetalen Fehlbildungen und anderen Marker untersucht (siehe Tab. 4):

\begin{tabular}{|l|l|}
\hline Anomalie & Beschreibung \\
\hline Softmarker & intrakardialer echogener Fokus (ICEF) \\
& Pyelektasie \\
singuläre Nabelschnurarterie (SUA) & echogener Darm \\
& Plexus-choroideus-Zysten (CPC) \\
& kurzes Femur / kurzer Humerus \\
& Ventrikulomegalie \\
\hline Fehlbildungen & Fehlbildungen des Zentralen Nervensystems (ZNS) \\
& Fehlbildungen der Niere \\
& Herzfehler \\
& Zwerchfellhernien \\
& Lippen-Kiefer-Gaumen-Spalte (LKG) \\
& Fehlbildungen der Bauchwand \\
& Fehlbildungen der Extremitäten \\
\hline Andere Marker & Hygrom \\
& Hydrops fetalis \\
& Aszites \\
& Hydro- / Chylothorax \\
& Perikarderguss \\
& Retardierung \\
& Polyhydramnion \\
& Oligohydramnion \\
& Anhydramnion \\
\hline
\end{tabular}

Tab. 4: Ultraschallanomalien, die für die vorliegende Studie untersucht wurden

Sowohl in Göttingen, als auch in Northeim wurden folgende Ultraschallgeräte verwendet:

- $\quad$ ATL HDI 3000

- $\quad$ ATL HDI 5000.

Untersucht wurde mit den Schallköpfen C5-2: dabei handelt es sich um konvex geformte Schallköpfe mit Frequenzen von 5 bis $2 \mathrm{MHz}$. 


\section{Ergebnisse}

\subsection{Altersverteilung der Patientinnen und Gestationswoche (SSW) zum Zeitpunkt der Amniozentese}

Im Zeitraum von 1993 bis 2003 wurden die in Göttingen und Northeim durchgeführten Amniozentesen $\geq 15$. SSW retrospektiv evaluiert. Dabei wurden die Amniozentesen bei den in dieser Arbeit vorgestellten 594 Schwangeren in der Zeit vom 12.01.1993 bis zum 23.07.2003 durchgeführt.

Das Alter konnte bei 590 (99,3 \%) der insgesamt 594 evaluierten Patientinnen ermittelt werden. Zum Zeitpunkt der jeweiligen Amniozentese betrug es durchschnittlich 29,1 Jahre ( $\mathrm{SD} \pm 5,2$, siehe Kapitel 9.1, S. 59). Die jüngste Patientin war 16, die älteste 46 Jahre alt.

Die mittlere Schwangerschaftsdauer zum Zeitpunkt der Amniozentese lag bei 22,7 SSW $(\mathrm{SD} \pm 5,4)$. Dabei wurde die früheste Punktion in der 15., die späteste in der 39. SSW vorgenommen.

Das Alter der Patientinnen mit positivem Softmarkerbefund betrug zum Zeitpunkt der Untersuchung im Mittel 29,8 Jahre (SD \pm 5,2). Auch hier reichte die Altersspanne von 16 bis 46 Jahre.

Die mittlere Schwangerschaftsdauer zum Zeitpunkt der Amniozentese lag bei diesen Patientinnen bei 21,7 SSW (SD $\pm 4,0$ ). Die früheste Punktion wurde hier in der 15., die späteste in der 37. SSW vorgenommen.

Das durchschnittliche Alter der Patientinnen mit fetalen Fehlbildungen und / oder anderen Markern betrug zum Zeitpunkt der Amniozentese 28,5 Jahre (SD $\pm 5,1$ ). Hier war die jüngste Patientin 16, die älteste 42 Jahre alt.

Die mittlere Schwangerschaftsdauer der Patientinnen mit fetalen Fehlbildungen und / oder anderen Markern lag zum Zeitpunkt der Amniozentese bei 23,5 SSW (SD \pm 6,1). Dabei reichte die Spanne von der 15. bis zur 39. SSW. 
Die Unterschiede zwischen den beiden Vergleichsgruppen bzgl. des durchschnittlichen mütterlichen Alters und der durchschnittlichen Schwangerschaftsdauer zum Zeitpunkt der Amniozentese werden in Tab. 5 dargestellt:

\begin{tabular}{|c|c|c|}
\hline & mütterliches Alter [Jahre] & Schwangerschaftsdauer [SSW] \\
\hline Softmarker & 29,8 & 21,7 \\
\hline $\begin{array}{c}\text { fetale Fehlbildungen und } \\
\text { andere Marker }\end{array}$ & 28,5 & 23,5 \\
\hline
\end{tabular}

Tab. 5: Unterschiede bzgl. des mittleren mütterlichen Alters und der mittleren Schwangerschaftsdauer

Die unterschiedliche Verteilung des mütterlichen Alters zeigt Abb. 8:

\section{Unterschiede beim mütterlichen Alter}

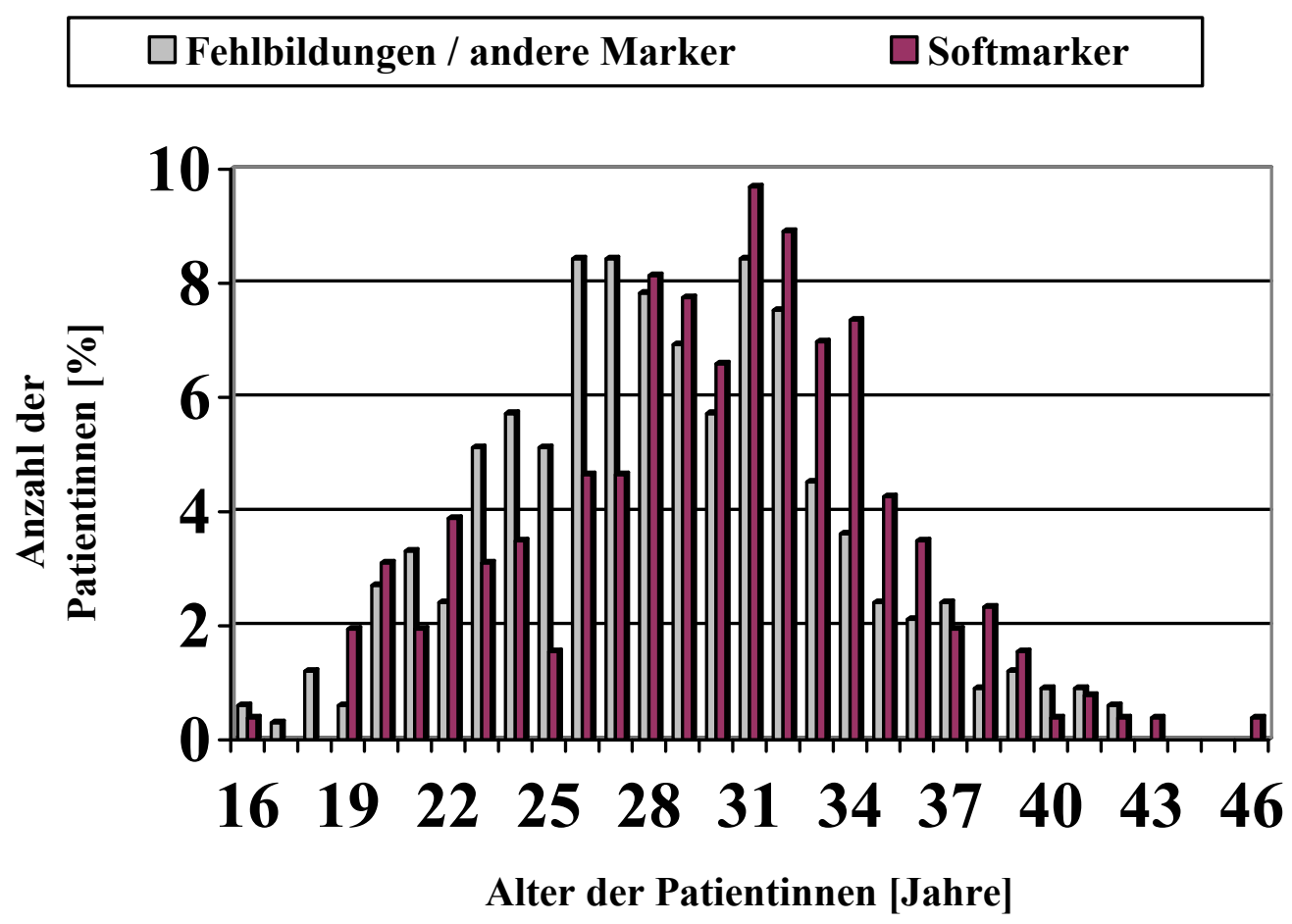

Abb. 8: Unterschiede in der Verteilung des mütterlichen Alters

Eine Amniozentese wurde bei Vorliegen von fetalen Fehlbildungen oder anderen Markern anders als bei Vorliegen von Softmarkern - häufig auch noch in späteren Schwangerschaftswochen durchgeführt. 


\subsection{Häufigkeit von Softmarkern}

\subsubsection{Allgemeines}

Insgesamt fanden sich Softmarker bei 303 der 594 untersuchten Feten $(51,0 \%)$ mit Auffälligkeiten im pränatalen Ultraschall.

Für diese Studie wurden allerdings nur 259 (85,5 \%) der 303 Schwangerschaften mit positivem Softmarkerbefund weiter verfolgt, da die übrigen $44(14,5 \%)$ Feten zusätzlich schwere Fehlbildungen aufwiesen, und die Indikation zur Amniozentese daher nicht allein aufgrund eines positiven Softmarkerbefundes gestellt wurde.

Daher wurden diese 44 Feten der Gruppe „fetale Fehlbildungen und andere Marker“ zugeteilt (siehe Kapitel 3.1, S. 20), und nur 259 (43,6 \%) der 594 Feten der Gruppe „Softmarker“ zugeordnet.

Tab. 6 veranschaulicht noch einmal, welche Softmarker für diese Studie untersucht wurden:

\begin{tabular}{|l|l|}
\hline Softmarker & intrakardialer echogener Fokus (ICEF) \\
& Pyelektasie \\
& singuläre Nabelschnurarterie (SUA) \\
& echogener Darm \\
& Plexus-choroideus-Zysten (CPC) \\
& kurzes Femur / kurzer Humerus \\
& Ventrikulomegalie \\
\hline
\end{tabular}

Tab. 6: Softmarker

Bei der Zusammenstellung der Häufigkeiten der verschiedenen Softmarker wurde jeder Softmarker einzeln erfasst.

Da bei einigen Feten allerdings mehrere Softmarker gleichzeitig vorlagen, ist die Summe der einzelnen Softmarker größer als die der betreffenden 259 Feten (s. u.). 
Insgesamt fanden sich also 284 Softmarker, da bei $234(90,3 \%)$ der 259 evaluierten Schwangeren ein isolierter Softmarker vorlag, wohingegen bei $25(9,7 \%)$ Patientinnen zwei Softmarker gleichzeitig auftraten (siehe Tab. 7).

\begin{tabular}{|c|c|c|c|}
\hline Softmarker & absolute Anzahl & $\begin{array}{c}\text { prozentualer Anteil } \\
\text { Softmarker [\%] }\end{array}$ & $\begin{array}{c}\text { prozentualer Anteil } \\
\text { gesamt [\%] }\end{array}$ \\
\hline ICEF & 12 & 4,2 & 2,0 \\
\hline Pyelektasie & 192 & 67,6 & 32,3 \\
\hline SUA & 15 & 5,3 & 2,5 \\
\hline echogener Darm & 1 & 0,4 & 0,2 \\
\hline CPC & 45 & 15,8 & 7,6 \\
\hline kurzes Femur / & 4 & 1,4 & 0,7 \\
\hline $\begin{array}{c}\text { Ventrikulomegalie } \\
\text { gesamt }\end{array}$ & 15 & 5,3 & 2,5 \\
\hline 284 & 100 & 47,8 \\
\hline
\end{tabular}

Tab. 7: Verteilung der einzelnen Softmarker

Mit einem Anteil von 67,6 \% (192 / 284) wurde weit über die Hälfte der Softmarker durch die Pyelektasie ausgemacht.

\subsubsection{Pyelektasie}

In unserem Studienkollektiv machte die Pyelektasie mit $67,6 \%$ den größten Anteil der auffälligen sonographischen Softmarker aus.

Insgesamt entdeckten wir eine Pyelektasie bei 192 der 594 untersuchten Feten. 110 (57,3\%) dieser 192 Feten waren männlich, 82 (42,7 \%) weiblich.

Das mittlere mütterliche Alter betrug in dieser Gruppe 30,1 Jahre (SD $\pm 5,1$ ), und die durchschnittliche Schwangerschaftsdauer lag zum Zeitpunkt der Amniozentese bei 21,5 SSW $(\mathrm{SD} \pm 3,7)$

Die Daten von 154 (80,2 \%) dieser Feten wurden in Northeim erhoben, diejenigen von 31 $(16,1 \%)$ Feten in Göttingen. Bei den übrigen 7 Feten war die Zuordnung zu einem Zentrum nicht eindeutig möglich. 


\subsubsection{Kombination von Softmarkern}

Tab. 8 veranschaulicht, welche Softmarker wie häufig in Kombination mit anderen Softmarkern auftraten.

Hierbei fällt auf, dass die intrakardialen echogenen Foci (ICEF) und das kurze Femur / der kurze Humerus prozentual am häufigsten mit anderen Softmarkern kombiniert waren, nämlich in jeweils der Hälfte der Fälle.

Dahingegen trat der echogene Darm überhaupt nicht in Kombination mit anderen Softmarkern auf.

\begin{tabular}{|c|c|c|c|c|c|c|c|}
\hline & ICEF & $\begin{array}{c}\text { Pyelek- } \\
\text { tasie }\end{array}$ & SUA & $\begin{array}{c}\text { echogen. } \\
\text { Darm }\end{array}$ & CPC & $\begin{array}{c}\text { Femur / } \\
\text { Humerus }\end{array}$ & $\begin{array}{c}\text { Ventriku- } \\
\text { lomegalie }\end{array}$ \\
\hline ICEF & --- & 6 & -- & --- & --- & --- & -- \\
\hline $\begin{array}{c}\text { Pyelek- } \\
\text { tasie }\end{array}$ & 6 & --- & 1 & --- & 10 & 2 & 4 \\
\hline SUA & --- & 1 & --- & --- & 2 & --- & --- \\
\hline $\begin{array}{c}\text { echogen. } \\
\text { Darm }\end{array}$ & --- & --- & -- & --- & --- & --- & --- \\
\hline CPC & --- & 10 & 2 & --- & --- & --- & --- \\
\hline $\begin{array}{c}\text { Femur / } \\
\text { Humerus }\end{array}$ & --- & 2 & --- & --- & --- & -- & - \\
\hline $\begin{array}{c}\text { Ventriku- } \\
\text { lomegalie }\end{array}$ & --- & 4 & -- & --- & -- & - \\
\hline
\end{tabular}

Tab. 8: Kombinationen von Softmarkern 


\subsection{Häufigkeit von fetalen Fehlbildungen und anderen Markern}

Der Gruppe „fetale Fehlbildungen und andere Marker“ wurden insgesamt 335 (56,4 \%) der 594 untersuchten Feten zugeordnet (siehe Kapitel 3.1, S. 20).

Fetale Fehlbildungen fanden sich bei 207 (34,8 \%), andere Marker bei 156 (26,3\%) aller Feten.

Da bei manchen Feten mehrere Fehlbildungen und / oder andere Marker gleichzeitig auftraten und die Auffälligkeiten bei einigen Feten nicht klassifiziert werden konnten (die daher in den folgenden Tabellen nicht erscheinen), stimmt die Summe der einzelnen Fehlbildungen und Marker nicht mit der Summe der 335 betroffenen Feten überein.

Tab. 9 veranschaulicht noch einmal, welche Anomalien unter den Begriffen „fetale Fehlbildungen“ und „,andere Marker“ zusammengefasst wurden:

\begin{tabular}{|l|l|}
\hline Fetale Fehlbildungen & Fehlbildungen des Zentralen Nervensystems (ZNS) \\
& Fehlbildungen der Nieren \\
& Herzfehler \\
& Zwerchfellhernien \\
& Lippen-Kiefer-Gaumen-Spalten (LKG) \\
& Fehlbildungen der Bauchwand \\
& Fehlbildungen der Extremitäten \\
\hline Andere Marker & Hygrom \\
& Hydrops fetalis \\
& Aszites \\
& Hydro- / Chylothorax \\
& Perikarderguss \\
& Retardierung \\
& Polyhydramnion \\
& Oligohydramnion \\
& Anhydramnion \\
\hline
\end{tabular}

Tab. 9: Fetale Fehlbildungen und andere Marker 
Klassifiziert werden konnten also 207 einzelne Fehlbildungen (siehe Tab. 10), wobei die Fehlbildungen des Zentralen Nervensystems (ZNS) in diesem Kollektiv mit 28,5 \% (59 / 207) den größten Anteil ausmachten.

\begin{tabular}{|c|c|c|c|}
\hline Fehlbildung & absolute Anzahl & $\begin{array}{c}\text { prozentualer Anteil } \\
\text { Fehlbildungen [\%] }\end{array}$ & $\begin{array}{c}\text { prozentualer Anteil } \\
\text { gesamt [\%] }\end{array}$ \\
\hline ZNS & 59 & 28,5 & 9,9 \\
\hline Nieren & 39 & 18,8 & 6,6 \\
\hline Herzfehler & 33 & 15,9 & 5,6 \\
\hline Zwerchfellhernie & 3 & 1,5 & 0,5 \\
\hline LKG-Spalte & 13 & 6,3 & 2,2 \\
\hline Bauchwand & 37 & 17,9 & 6,2 \\
\hline Extremitäten & 23 & 11,1 & 3,9 \\
\hline gesamt & 207 & 100 & 34,9 \\
\hline
\end{tabular}

Tab. 10: Verteilung der einzelnen Fehlbildungen 
Bei den anderen Markern konnten 174 einzelne Marker klassifiziert werden (siehe Tab. 11).

\begin{tabular}{|c|c|c|c|}
\hline andere Marker & absolute Anzahl & $\begin{array}{c}\text { prozentualer Anteil } \\
\text { andere Marker [\%] }\end{array}$ & $\begin{array}{c}\text { prozentualer Anteil } \\
\text { gesamt [\%] }\end{array}$ \\
\hline Hygrom & 18 & 10,3 & 3,0 \\
\hline Hydrops fetalis & 5 & 2,9 & 0,8 \\
\hline Aszites & 6 & 3,5 & 1,0 \\
\hline Hydro- / Chylothorax & 3 & 1,7 & 0,5 \\
\hline Perikarderguss & 2 & 1,2 & 0,3 \\
\hline Retardierung & 56 & 32,2 & 9,4 \\
\hline Polyhydramnion & 39 & 22,4 & 6,6 \\
\hline Oligohydramnion & 38 & 21,8 & 6,4 \\
\hline Anhydramnion & 7 & 4,0 & 1,2 \\
\hline gesamt & 174 & 100 & 29,3 \\
\hline
\end{tabular}

Tab. 11: Verteilung der einzelnen Marker

Unter diesen machten die Abweichungen in der Fruchtwassermenge (Poly-, Oligo- und Anhydramnion) mit insgesamt 48,3 \% (84 / 174) den größten Teil aus. 
Die Tab. 12, Tab. 13 und Tab. 14 veranschaulichen, wie häufig die einzelnen Fehlbildungen und anderen Marker in Kombination miteinander auftraten.

\begin{tabular}{|c|c|c|c|c|c|c|c|c|}
\hline & \multicolumn{7}{|c|}{ Fehlbildungen } \\
\hline & & ZNS & Niere & Herz & $\begin{array}{l}\text { Zwerch- } \\
\text { fell }\end{array}$ & LKG & $\begin{array}{l}\text { Bauch- } \\
\text { wand }\end{array}$ & $\begin{array}{c}\text { Extremi- } \\
\text { täten }\end{array}$ \\
\hline \multirow{7}{*}{ 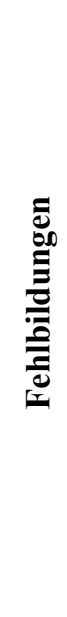 } & ZNS & --- & 1 & 2 & --- & 2 & 2 & 4 \\
\hline & Niere & 1 & --- & 1 & 1 & 2 & --- & --- \\
\hline & Herz & 2 & 1 & --- & --- & 3 & 3 & 4 \\
\hline & $\begin{array}{c}\text { Zwerch- } \\
\text { fell }\end{array}$ & --- & 1 & --- & --- & --- & --- & --- \\
\hline & LKG & 2 & 2 & 3 & --- & --- & 1 & 2 \\
\hline & $\begin{array}{l}\text { Bauch- } \\
\text { wand }\end{array}$ & 2 & --- & 3 & --- & 1 & --- & 3 \\
\hline & $\begin{array}{c}\text { Extremi- } \\
\text { täten }\end{array}$ & 4 & --- & 4 & --- & 2 & 3 & --- \\
\hline
\end{tabular}

Tab. 12: Kombinationen von Fehlbildungen untereinander

\begin{tabular}{|c|c|c|c|c|c|c|c|c|}
\hline & & \multicolumn{7}{|c|}{ Fehlbildungen } \\
\hline & & ZNS & Niere & Herz & $\begin{array}{c}\text { Zwerch- } \\
\text { fell }\end{array}$ & LKG & $\begin{array}{c}\text { Bauch- } \\
\text { wand }\end{array}$ & $\begin{array}{c}\text { Extremi- } \\
\text { täten }\end{array}$ \\
\hline \multirow{9}{*}{ 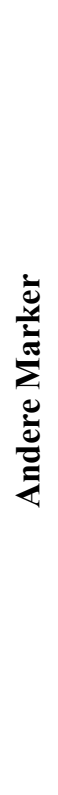 } & Hygrom & --- & --- & --- & --- & --- & 1 & 1 \\
\hline & Hydrops & --- & --- & --- & --- & --- & 1 & --- \\
\hline & Aszites & --- & --- & --- & --- & --- & --- & --- \\
\hline & $\begin{array}{l}\text { Hydro- } \\
\text { thorax }\end{array}$ & --- & --- & --- & --- & --- & --- & --- \\
\hline & Perikard & --- & --- & 1 & --- & --- & 1 & --- \\
\hline & $\begin{array}{l}\text { Retar- } \\
\text { dierung }\end{array}$ & 1 & 1 & 7 & --- & 2 & --- & 2 \\
\hline & $\begin{array}{l}\text { Polyhy- } \\
\text { dramn. }\end{array}$ & 3 & 2 & 3 & 1 & 1 & 4 & 3 \\
\hline & $\begin{array}{l}\text { Oligohy- } \\
\text { dramn. }\end{array}$ & 2 & 5 & 1 & --- & --- & --- & --- \\
\hline & $\begin{array}{l}\text { Anhy- } \\
\text { dramn. }\end{array}$ & --- & 1 & --- & --- & --- & --- & 1 \\
\hline
\end{tabular}

Tab. 13: Kombinationen von Fehlbildungen und anderen Markern 
Störungen des ZNS und Bauchwanddefekte führen aufgrund eines gestörten Schluckaktes häufig zu einer vermehrten Fruchtwassermenge i. S. e. Polyhydramnion, während Fehlbildungen in den ableitenden Harnwegen zu einem Oligohydramnion führen.

\begin{tabular}{|c|c|c|c|c|c|c|c|c|c|c|}
\hline & \multicolumn{9}{|c|}{ Andere Marker } \\
\hline & & $\begin{array}{c}\text { Hy- } \\
\text { grom }\end{array}$ & $\begin{array}{c}\text { Hy- } \\
\text { drops }\end{array}$ & $\begin{array}{c}\text { Aszi- } \\
\text { tes }\end{array}$ & $\begin{array}{l}\text { Hydro- } \\
\text { thorax }\end{array}$ & $\begin{array}{l}\text { Peri- } \\
\text { kard }\end{array}$ & $\begin{array}{l}\text { Retar- } \\
\text { dier. }\end{array}$ & $\begin{array}{l}\text { Poly- } \\
\text { hydr. }\end{array}$ & $\begin{array}{l}\text { Oligo- } \\
\text { hydr. }\end{array}$ & $\begin{array}{l}\text { An- } \\
\text { hydr. }\end{array}$ \\
\hline \multirow{9}{*}{ 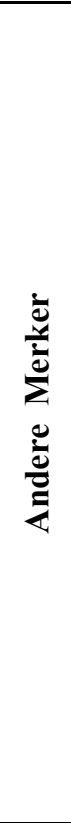 } & $\begin{array}{c}\text { Hy- } \\
\text { grom }\end{array}$ & --- & 3 & --- & 1 & --- & 1 & --- & --- & --- \\
\hline & $\begin{array}{c}\text { Hy- } \\
\text { drops }\end{array}$ & 3 & --- & --- & --- & --- & --- & --- & --- & --- \\
\hline & $\begin{array}{c}\text { Aszi- } \\
\text { tes }\end{array}$ & --- & --- & --- & --- & --- & --- & --- & --- & --- \\
\hline & $\begin{array}{l}\text { Hydro- } \\
\text { thorax }\end{array}$ & 1 & --- & --- & --- & --- & 1 & 1 & --- & --- \\
\hline & $\begin{array}{l}\text { Peri- } \\
\text { kard }\end{array}$ & --- & --- & --- & --- & --- & --- & 1 & --- & --- \\
\hline & $\begin{array}{c}\text { Retar- } \\
\text { dier. }\end{array}$ & 1 & --- & --- & 1 & --- & --- & 3 & 15 & 2 \\
\hline & $\begin{array}{l}\text { Poly- } \\
\text { hydr. }\end{array}$ & --- & --- & --- & 1 & 1 & 3 & --- & --- & --- \\
\hline & $\begin{array}{l}\text { Oligo- } \\
\text { hydr. }\end{array}$ & --- & --- & --- & --- & --- & 15 & --- & --- & --- \\
\hline & $\begin{array}{c}\text { An- } \\
\text { hydr. }\end{array}$ & --- & --- & --- & --- & --- & 2 & --- & --- & --- \\
\hline
\end{tabular}

Tab. 14. Kombinationen der anderen Marker untereinander

Darüber hinaus zeigt Tab. 14, dass das Oligohydramnion in einem hohen Maße mit einer fetalen Retardierung assoziiert ist. Hier verweisen wir auch auf eine Vorarbeit der Göttinger Arbeitsgruppe, die den Zusammenhang zwischen Abweichungen in der Fruchtwassermenge und assoziierten fetalen Störungen untersuchte (PAUER et al. 2003). 


\subsection{Häufigkeit von Chromosomenanomalien}

In dem Gesamtkollektiv der 594 untersuchten Schwangerschaften fanden sich Chromosomenanomalien bei $63(10,6 \%)$ Feten.

Diese beinhalteten sowohl numerische als auch strukturelle Aberrationen (siehe Tab. 15):

\begin{tabular}{|c|c|c|c|}
\hline $\begin{array}{c}\text { Chromosomen- } \\
\text { anomalie }\end{array}$ & absolute Anzahl & $\begin{array}{c}\text { prozentualer Anteil } \\
\text { Anomalien [\%] }\end{array}$ & $\begin{array}{c}\text { prozentualer Anteil } \\
\text { gesamt [\%] }\end{array}$ \\
\hline $\begin{array}{c}\text { Trisomie 21 } \\
\text { (DowN-Syndrom) }\end{array}$ & 17 & 27,0 & 2,9 \\
\hline $\begin{array}{c}\text { Trisomie 18 } \\
\text { (EDWARDS-Syndrom) }\end{array}$ & 16 & 25,4 & 2,7 \\
\hline $\begin{array}{c}\text { Trisomie 13 } \\
\text { (PÄTAU-Syndrom) }\end{array}$ & 7 & 11,1 & 1,2 \\
\hline $\begin{array}{c}\text { Monosomie X } \\
\text { (TuRNER-Syndrom) }\end{array}$ & 6 & 9,5 & 1,0 \\
\hline Triploidie & 7 & 11,1 & 1,2 \\
\hline andere & 10 & 15,9 & 1,7 \\
\hline gesamt & 63 & 100 & 10,6 \\
\hline
\end{tabular}

Tab. 15: Verteilung der Chromosomenanomalien im Gesamtkollektiv

Bei den Trisomien 21, 18 und 13, sowie bei der Monosomie $\mathrm{X}$ handelt es sich um numerische Chromosomenanomalien, d. h. die betroffenen Feten weisen nicht 46, sondern 47 bzw. nur 45 Chromosomen auf.

Von einer Triploidie spricht man, wenn ein Fet drei haploide Chromosomensätze aufweist: statt der üblichen 46 besitzt er 69 Chromosomen.

Unter dem Begriff ,andere“ wurden für die vorliegende Arbeit strukturelle Chromosomenanomalien wie z. B. Deletionen, Inversionen oder Translokationen zusammen gefasst. Hierbei handelt es sich nicht um Abweichungen in der Anzahl der Chromosomen, sondern um strukturelle Veränderungen einzelner Chromosomenabschnitte.

Mit 27,0 \% (17 / 63) hatte die Trisomie 21 (Down-Syndrom) den größten Anteil unter den Chromosomenanomalien, dicht gefolgt von der Trisomie 18 (EDWARDS-Syndrom) mit 25,4 \% $(16 / 63)$. 
In der Gruppe der Patientinnen mit einem positiven Softmarkerbefund fanden sich Chromosomenanomalien bei 10 (3,9\%) der 259 Feten (siehe Tab. 16).

\begin{tabular}{|c|c|c|c|}
\hline $\begin{array}{c}\text { Chromosomen- } \\
\text { anomalie }\end{array}$ & absolute Anzahl & $\begin{array}{c}\text { prozentualer Anteil } \\
\text { Anomalien [\%] }\end{array}$ & $\begin{array}{c}\text { prozentualer Anteil } \\
\text { gesamt [\%] }\end{array}$ \\
\hline $\begin{array}{c}\text { Trisomie 21 } \\
\text { (DowN-Syndrom) }\end{array}$ & 5 & 50,0 & 1,9 \\
\hline $\begin{array}{c}\text { Trisomie 18 } \\
\text { (EDWARDS-Syndrom) }\end{array}$ & 2 & 20,0 & 0,8 \\
\hline $\begin{array}{c}\text { Trisomie 13 } \\
\text { (PäTAU-Syndrom) }\end{array}$ & --- & --- & -- \\
\hline $\begin{array}{c}\text { Monosomie X } \\
\text { (TURNER-Syndrom) }\end{array}$ & --- & -- & --- \\
\hline Triploidie & 1 & 10,0 & 0,4 \\
\hline andere & 2 & 20,0 & 0,8 \\
\hline gesamt & 10 & 100 & 3,9 \\
\hline
\end{tabular}

Tab. 16: Verteilung der Chromosomenanomalien bei Patientinnen mit positivem Softmarkerbefund

Bei allen 10 Feten, die Chromosomenanomalien aufwiesen, lagen die Softmarker isoliert vor. Von den 25 Feten, bei denen zwei Softmarker gleichzeitig auftraten, litt dahingegen keiner an einer Chromosomenanomalie.

In dieser Gruppe machte die Trisomie 21 mit 50,0 \% (5 / 10) den weitaus größten Teil aus, wohingegen sowohl die Trisomie 13 (PÄTAU-Syndrom), als auch die Monosomie X (TURNERSyndrom) gar nicht vertreten waren. 
Tab. 17 gibt einen Überblick über die Charakteristika dieser 10 von einer Chromosomenanomalie betroffenen Feten mit positivem Softmarkerbefund:

\begin{tabular}{|c|c|c|c|c|c|}
\hline $\begin{array}{l}\text { Chromosomen- } \\
\text { anomalie }\end{array}$ & Karyotyp & Softmarker & $\begin{array}{l}\text { mütterliches } \\
\text { Alter }\end{array}$ & SSW & Zentrum \\
\hline \multirow{5}{*}{ Trisomie 21} & $47, X Y+21$ & \multirow{4}{*}{ Pyelektasie } & 39 & 24 & NOM \\
\hline & $47, \mathrm{XY}+21$ & & 29 & 16 & NOM \\
\hline & $47, X X+21$ & & 20 & 31 & GÖ \\
\hline & $47, X X+21$ & & 20 & 17 & NOM \\
\hline & $47, X X+21$ & SUA & 33 & 18 & GÖ \\
\hline \multirow{2}{*}{ Trisomie 18} & $47, X X+18$ & \multirow{2}{*}{$\mathrm{CPC}$} & 28 & 20 & $\mathrm{NOM}$ \\
\hline & $47, \mathrm{XY}+18$ & & 21 & 18 & NOM \\
\hline Triploidie & $69, \mathrm{XXX}$ & $\begin{array}{l}\text { Ventrikulo- } \\
\text { megalie }\end{array}$ & 36 & 17 & NOM \\
\hline \multirow{2}{*}{ andere } & 46,XX,add(8)(p23) & \multirow{2}{*}{ CPC } & 31 & 21 & NOM \\
\hline & $46, X Y, \operatorname{add}(14)(\mathrm{p} 11)$ & & 19 & 22 & GÖ \\
\hline
\end{tabular}

Tab. 17: Feten mit einer Chromosomenanomalie und positivem Softmarkerbefund 
In der Gruppe „fetale Fehlbildungen und andere Marker“ fanden sich Chromosomenanomalien bei 51 (15,2\%) der 335 Feten (siehe Tab. 18).

\begin{tabular}{|c|c|c|c|}
\hline $\begin{array}{c}\text { Chromosomen- } \\
\text { anomalie }\end{array}$ & absolute Anzahl & $\begin{array}{c}\text { prozentualer Anteil } \\
\text { Anomalien [\%] }\end{array}$ & $\begin{array}{c}\text { prozentualer Anteil } \\
\text { gesamt [\%] }\end{array}$ \\
\hline $\begin{array}{c}\text { Trisomie 21 } \\
\text { (DowN-Syndrom) }\end{array}$ & 12 & 23,5 & 3,6 \\
\hline $\begin{array}{c}\text { Trisomie 18 } \\
\text { (EDWARDS-Syndrom) }\end{array}$ & 14 & 27,5 & 4,2 \\
\hline $\begin{array}{c}\text { Trisomie 13 } \\
\text { (PÄTAU-Syndrom) }\end{array}$ & 7 & 13,7 & 2,1 \\
\hline $\begin{array}{c}\text { Monosomie X } \\
\text { (TURNER-Syndrom) }\end{array}$ & 6 & 11,8 & 1,8 \\
\hline Triploidie & 6 & 11,8 & 1,8 \\
\hline andere & 6 & 11,8 & 1,8 \\
\hline gesamt & 51 & 100 & 15,2 \\
\hline
\end{tabular}

Tab. 18: Verteilung der Chromosomenanomalien in der Gruppe „fetale Fehlbildungen und andere Marker“

In dieser Gruppe hatte die Trisomie 18 (EDWARDS-Syndrom) mit 27,5 \% (14 / 51) den größten Anteil, gefolgt von der Trisomie 21 (Down-Syndrom) mit 23,5 \% (12 / 51). 
Die Unterschiede in der Verteilung der Chromosomenanomalien in den unterschiedlichen Vergleichsgruppen werden in $A b b .9$ graphisch dargestellt.

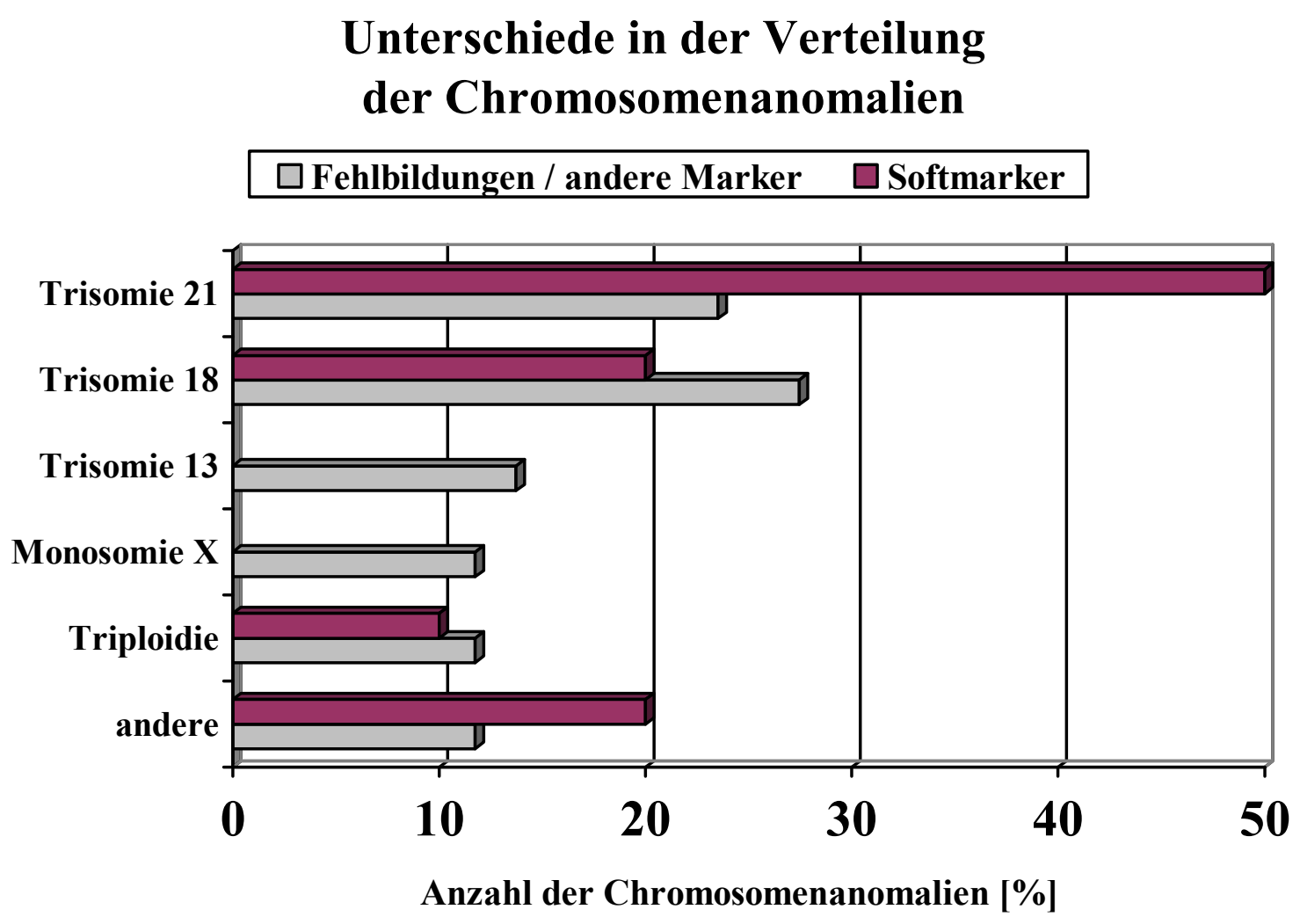

Abb. 9: Unterschiede in der Verteilung der Chromosomenanomalien 


\subsection{Häufigkeit von Chromosomenanomalien bei positivem Softmarkerbefund, abhängig vom mütterlichen Alter}

Das erhöhte mütterliche Alter ( $\geq 35$ Jahre) ist ein Risikofaktor für das Auftreten einer fetalen

Chromosomenanomalie.

Um auszuschließen, dass das Fehlbildungsrisiko der für diese Studie untersuchten Feten mit positivem Softmarkerbefund allein aufgrund dieses mütterlichen Risikofaktors beeinflusst wurde, sind die Fehlbildungsraten der Schwangeren $\geq 35$ Jahre denjenigen der Patientinnen $<35$ Jahre gegenüber gestellt worden.

Da das Alter bei einer Schwangeren nicht evaluiert werden konnte, zeigt Tab. 19 nur die Daten von 258 Patientinnen:

\begin{tabular}{|c|c|c|c|c|c|c|}
\hline \multirow{2}{*}{$\begin{array}{c}\text { Chromo- } \\
\text { somen- } \\
\text { anomalie }\end{array}$} & \multicolumn{3}{|c|}{ Patientinnen } \\
\cline { 2 - 7 } & absolut & $\begin{array}{c}\text { prozentual } \\
<\mathbf{3 5} \text { Jahre }\end{array}$ & $\begin{array}{c}\text { prozentual } \\
\text { gesamt }\end{array}$ & absolut & $\begin{array}{c}\text { prozentual } \\
\geq \mathbf{3 5} \text { Jahre }\end{array}$ & $\begin{array}{c}\text { prozentual } \\
\text { gesamt }\end{array}$ \\
\cline { 2 - 7 } ja & 8 & 3,7 & 3,1 & 2 & 4,9 & 0,8 \\
\hline nein & 209 & 96,3 & 81,0 & 39 & 95,1 & 15,1 \\
\hline gesamt & 217 & 100 & 84,1 & 41 & 100 & 15,9 \\
\hline
\end{tabular}

Tab. 19: Häufigkeit von Chromosomenanomalien in Abhängigkeit vom mütterlichen Alter

Unter den Schwangeren mit positivem Softmarkerbefund, die zum Punktionszeitpunkt $\geq 35$ Jahre alt waren, lag bei 4,9\% eine fetale Chromosomenanomalie vor.

Bei den Schwangeren $<35$ Jahre lag die Häufigkeit für eine Chromosomenanomalie bei $3,7 \%$.

Somit besteht in diesem Kollektiv kein signifikanter Unterschied zwischen den beiden Altersgruppen hinsichtlich der Rate fetaler Chromosomenanomalien (siehe Kapitel 9.2.1, S. 59). 


\subsection{Häufigkeit von Chromosomenanomalien bei Vorliegen eines isolierten Softmarkers}

\subsubsection{Pyelektasie}

Von den 169 Feten mit einer isoliert auftretenden Pyelektasie wiesen 4 (2,4 \%) eine Chromosomenanomalie auf (siehe Tab. 20).

\begin{tabular}{|c|c|c|c|}
\hline \multirow{2}{*}{$\begin{array}{c}\text { Chromosomen- } \\
\text { anomalie }\end{array}$} & \multicolumn{3}{|c|}{ mit milder Pyelektasie } \\
\cline { 2 - 4 } & absolute Anzahl & $\begin{array}{c}\text { prozentualer Anteil } \\
\text { Anomalien [\%] }\end{array}$ & $\begin{array}{c}\text { prozentualer Anteil } \\
\text { gesamt [\%] }\end{array}$ \\
\hline $\begin{array}{c}\text { Trisomie 21 } \\
\text { (Down-Syndrom) }\end{array}$ & 4 & 100,0 & 2,4 \\
\hline $\begin{array}{c}\text { Trisomie 18 } \\
\text { (EDWARDS-Syndrom) }\end{array}$ & --- & --- & - --- \\
\hline $\begin{array}{c}\text { Trisomie 13 } \\
\text { (PäTAU-Syndrom) }\end{array}$ & --- & --- & -- \\
\hline $\begin{array}{c}\text { Monosomie X } \\
\text { (TURNER-Syndrom) }\end{array}$ & --- & --- & - \\
\hline Triploidie & --- & --- & \\
\hline andere & --- & 100 & 2,4 \\
\hline
\end{tabular}

Tab. 20: Häufigkeit von Chromosomenanomalien bei Feten mit einer Pyelektasie

Von diesen 4 Feten waren 2 weiblich und 2 männlich. Dies bedeutet, dass 2,9 \% (2 / 70) der weiblichen und von einer isolierten Pyelektasie betroffenen Feten eine Chromosomenanomalie aufwiesen, verglichen mit 2,0 \% (2/99) der männlichen Feten. 


\subsubsection{Plexus-choroideus-Zysten (CPC)}

Von den 33 Feten mit isolierten Plexus-choroideus-Zysten (CPC) wiesen 4 (12,1\%) eine Chromosomenanomalie auf (siehe Tab. 21).

\begin{tabular}{|c|c|c|c|}
\hline \multirow{2}{*}{$\begin{array}{l}\text { Chromosomen- } \\
\text { anomalie }\end{array}$} & \multicolumn{3}{|c|}{ mit CPC } \\
\hline & absolute Anzahl & $\begin{array}{c}\text { prozentualer Anteil } \\
\text { Anomalien [\%] }\end{array}$ & $\begin{array}{c}\text { prozentualer Anteil } \\
\text { gesamt [\%] }\end{array}$ \\
\hline $\begin{array}{c}\text { Trisomie } 21 \\
\text { (Down-Syndrom) }\end{array}$ & --- & --- & --- \\
\hline $\begin{array}{c}\text { Trisomie } 18 \\
\text { (EDWARDS-Syndrom) }\end{array}$ & 2 & 50,0 & 6,1 \\
\hline $\begin{array}{c}\text { Trisomie 13 } \\
\text { (PäTAU-Syndrom) }\end{array}$ & --- & --- & --- \\
\hline $\begin{array}{c}\text { Monosomie X } \\
\text { (TURNER-Syndrom) } \\
\end{array}$ & --- & --- & --- \\
\hline Triploidie & --- & --- & --- \\
\hline andere & 2 & 50,0 & 6,1 \\
\hline gesamt & 4 & 100 & 12,1 \\
\hline
\end{tabular}

Tab. 21: Häufigkeit von Chromosomenanomalien bei Feten mit CPC

\subsubsection{Ventrikulomegalie}

Einer der 11 Feten mit einer isoliert auftretenden Ventrikulomegalie wies eine Triploidie auf.

Die übrigen 10 Feten zeigten keine chromosomalen Auffälligkeiten.

\subsubsection{Kurzes Femur / kurzer Humerus}

Keiner der 4 Feten mit einem isolierten kurzen Femur / kurzen Humerus wies eine Chromosomenanomalie auf.

\subsubsection{Intrakardialer echogener Fokus (ICEF)}

Unter den 6 Feten mit isolierten intrakardialen echogenen Foci (ICEF) fand sich keiner mit einer Chromosomenanomalie. 


\subsubsection{Singuläre Nabelschnurarterie (SUA)}

Unter den 12 Feten mit einer isolierten SUA fand sich einer mit einer Trisomie 21 (DowN-

Syndrom). Die übrigen 11 Feten wiesen keine chromosomalen Auffälligkeiten auf.

\subsubsection{Echogener Darm}

Der einzige Fet, bei dem im pränatalen Ultraschall ein isoliert auftretender echogener Darm entdeckt worden war, wies keine Chromosomenanomalie auf. 


\subsubsection{Erläuterung}

Die der vorliegenden Arbeit zugrunde liegende Studie wurde im Jahr 1993 in dem Bestreben initiiert, das mit dem Auftreten von sonographischen Softmarkern einhergehende Risiko für das Vorliegen einer fetalen Chromosomenanomalie zu bestimmen. Zu diesem Zweck wurden die über einen Zeitraum von zehn Jahren an zwei pränatalmedizinischen Zentren erhobenen Daten zu sieben verschiedenen Softmarkern retrospektiv evaluiert.

Es erfolgte die Auswertung der Ultraschallprotokolle von Schwangeren, bei denen allein aufgrund des Vorliegens eines oder mehrerer sonographischer Softmarker eine Amniozentese durchgeführt wurde.

Auf der Grundlage dieser Protokolle wurden die folgenden Details erfasst:

- Anzahl der vorliegenden Softmarker (isoliert / multipel)

- Art des / der vorliegenden Softmarker

- Vorliegen einer fetalen Chromosomenanomalie

- Art der fetalen Chromosomenanomalie.

Nach Abschluss der Studie im Jahr 2003 zeigte sich allerdings, dass nur zu zwei der oben vorgestellten sieben Softmarker - nämlich zur Pyelektasie und den Plexus-choroideus-Zysten (CPC) - ausreichende Datenmengen akquiriert werden konnten, um eine verbindliche Aussage über das mit ihrem Auftreten assoziierten Risiko für eine fetale Chromosomenanomalie treffen zu können.

Um zu erfahren, warum die übrigen fünf Softmarker z. T. nur sehr geringe Fallzahlen aufwiesen, wurde Rücksprache mit denjenigen Gynäkologen gehalten, welche damals die Ultraschalluntersuchungen bei den an der Studie teilnehmenden Schwangeren durchgeführt hatten.

Hierbei stellte sich heraus, dass die Gründe für die stark variierenden Fallzahlen (z. B. 192 Feten mit Pyelektasie gegenüber nur 12 Feten mit intrakardialen echogenen Foci (ICEF)) vor allem in der unterschiedlichen Gewichtung der einzelnen Softmarker durch die untersuchenden Ärzte lag.

Der Befund eines ICEF oder einer Pyelektasie führte an der Universitätsfrauenklinik Göttingen nach ausführlicher Aufklärung über das geringe Risiko für eine Chromosomenaberration häufiger zu einer seriellen Kontrolle der Feindiagnostik und seltener zu einer invasiven Diagnostik. Das Fehlen jeglicher Auffälligkeiten in der weiterführenden Ultraschall-Untersuchung ist mit einer Risikoverminderung um über die Hälfte verbunden (SOHL et al. 1999). 
Diese unterschiedliche Vorgehensweise bezüglich der verschiedenen Softmarker führte in den beiden Zentren folglich zu den z. T. recht stark variierenden Fallzahlen und erklärt die niedrige Prävalenz z. B. für die ICEF in unserem Studienkollektiv.

Aus diesem Grund liegt der Schwerpunkt in der sich anschließenden Diskussion auf den beiden Softmarkern Pyelektasie und Plexus-choroideus-Zysten. Die Fallzahlen der restlichen Softmarker scheinen keine ausreichende Grundlage zu bilden, um hier erkenntnisfördernd diskutiert zu werden.

Zusätzlich muss stets bedacht werden, dass die in den beiden teilnehmenden pränatalmedizinischen Zentren betreuten Schwangeren kein Normalkollektiv darstellen, sondern dass bei all diesen Patientinnen bereits im Vorfeld Auffälligkeiten festgestellt worden sind, welche zur Überweisung der Schwangeren an ein spezialisiertes Zentrum geführt haben.

Somit handelt es sich bei der in dieser Arbeit vorgestellten Patientengruppe um ein stark vorselektiertes Kollektiv, in dem alle Feten eine sonographisch diagnostizierbare Auffälligkeit aufwiesen. 


\section{Diskussion}

\subsection{Assoziation von sonographischen Softmarkern mit fetalen Chromosomenanomalien}

Als sonographische Softmarker bezeichnet man geringgradige Strukturauffälligkeiten in der Anatomie des Feten, welche zu einem gewissen Prozentsatz mit dem Auftreten fetaler Chromosomenanomalien assoziiert sind.

Anders als die sonographischen Hardmarker sind die Softmarker keine schwerwiegenden anatomischen Malformationen, welche auf ein spezifisches Fehlbildungssyndrom hinweisen können (BATUKAN et al. 2001), sondern lediglich ein Indikator für möglicherweise vorliegende fetale Anomalien.

Diese Eigenschaft macht die sonographischen Softmarker einerseits zu einem wertvollen diagnostischen Parameter in der Pränatalmedizin, andererseits verlangt ihre Entdeckung den werdenden Eltern wie auch den behandelnden Gynäkologien die schwere Entscheidung ab, ob und wann nach ihrer Entdeckung weitere - und damit ggf. auch invasive - diagnostische Maßnahmen folgen sollten.

Während verschiedene Studiengruppen (ChristinA et al. 2005, BETTELHEIM et al. 1999, TOMLINSON et al. 1997, KUPFERMINC et al. 1994) empfehlen, der Entdeckung sonographischer Softmarker unbedingt eine weitere Fehlbildungsdiagnostik folgen zu lassen, sehen andere z. B. in den ICEF lediglich Normvarianten im Zuge der fetalen Entwicklung (BRADLEY et al. 2005, PETRIKOVSKY et al. 1995).

Diese zum Teil sehr widersprüchlichen Aussagen zum weiteren Procedere nach der Diagnose von sonographischen Softmarkern führen zu einer beträchtlichen Verunsicherung bei den betroffenen Schwangeren und den sie beratenden Ärzten und Humangenetikern.

In dem Bestreben, sich selbst ein Bild zu machen und damit einen Beitrag zu der allgemeinen Diskussion liefern zu können, wurde die dieser Arbeit zugrunde liegende Studie initiiert.

Über einen Zeitraum von zehn Jahren wurden die Sonographieprotokolle und die Ergebnisse der dazugehörigen fetalen Chromosomenanalysen nach Amniozentese von insgesamt 594 Schwangeren erfasst und retrospektiv ausgewertet.

Das Ziel hierbei war es, die Assoziationsraten der einzelnen Softmarker mit fetalen Chromosomenaberrationen zu bestimmen, um den diagnostischen Wert der sonographischen Softmarker besser einschätzen und die betroffenen Schwangeren entsprechend besser beraten zu können. 
Alle Patientinnen, die in diese Studie eingeschlossen wurden, waren aufgrund einer im Ultraschall diagnostizierten fetalen Auffälligkeit von ihren behandelnden Gynäkologen an eines der beiden von uns betreuten Zentren für Pränatalmedizin (Universitätsfrauenklinik Göttingen bzw. Frauenklinik Northeim) überwiesen worden. Zusätzlich wurde bei allen teilnehmenden Schwangeren eine Amniozentese vorgenommen und das Ergebnis der hierbei gewonnenen fetalen Chromosomenanalyse mit erfasst.

Für die Auswertung der erhobenen Daten wurden die Patientinnen zunächst in zwei Gruppen aufgeteilt. Die Zuordnung der Frauen zu einer der beiden Studiengruppen erfolgte nach der sonographischen Auffälligkeit, welche zur Indikationsstellung für die Durchführung einer Amniozentese geführt hatte.

Auf diese Weise ergab sich ein Kollektiv $(n=259)$, in dem die Feten ausschließlich sonographische Softmarker aufwiesen, und ein Kollektiv $(n=335)$, bei dem eine Amniozentese aufgrund von echten fetalen Fehlbildungen und / oder sog. anderen Markern vorgenommen worden war.

Hierbei wird deutlich, dass für die vorliegende Studie kein die Allgemeinbevölkerung repräsentierendes Normalkollektiv untersucht wurde, sondern dass alle beobachteten Feten eine sonographische Auffälligkeit aufwiesen. Somit handelt es sich hier um ein vorselektiertes Patientenkollektiv, wie es für ein pränatalmedizinisches Zentrum typisch ist.

Wir fanden eine Chromosomenanomalie bei 3,9 \% (10/259) der Feten mit einem positiven Softmarkerbefund; bei all diesen Feten lagen die Softmarker isoliert vor. Die mit 50,0 \% (5 / 10) am häufigsten vertretene Aberration war die Trisomie 21 (Down-Syndrom).

Das Hintergrundrisiko einer 29jährigen Frau, ein Kind mit einer Chromosomenaberration zu bekommen, beträgt $0,24 \%$. Hiervon entfallen $0,1 \%$ auf die Trisomie 21 (Down-Syndrom) (SNIJDERS et al. 1999).

In dem von uns untersuchten Kollektiv der Feten mit positivem Softmarkerbefund, in dem das mittlere mütterliche Alter 29,8 Jahre betrug, liegt die Anomalie mit 3,9 \% rund 16mal höher, als das altersabhängige Hintergrundrisiko vermuten ließe.

Auch nach einer Studie von SoHL et al. (1999) erhöht die Entdeckung sonographischer Softmarker das Risiko für eine fetale Chromosomenanomalie deutlich: bei isolierten Softmarkern steigt es um das 5,7fache, bei multiplen Softmarkern erhöht es sich sogar um das 12fache gegenüber dem entsprechenden Risiko bei einer vollkommen unauffälligen Ultraschalluntersuchung. 
KURJAK et al. (1999) fanden ebenfalls erhöhte Anomalieraten: in ihrem Studienkollektiv wiesen 11,6 \% (7 / 60) der Feten mit isolierten und 14,2 \% $(23 / 162)$ der Feten mit multiplen Softmarkern einen abnormen Karyotyp auf. In ihrer Studie lag das mittlere mütterliche Alter bei 27 Jahren.

Ebenso fanden PARRA-CORDERO et al. (2007), welche insgesamt 3071 Frauen untersuchten, mindestens einen von vier Softmarkern und / oder kardialen Defekten bei 77,8 \% der Feten mit einem Down-Syndrom, verglichen mit lediglich 3,1 \% der Feten in der Kontrollgruppe. In dem von uns untersuchten gesamten Studienkollektiv $(n=594)$ wiesen 10,6 \% $(63 / 594)$ der Feten eine Chromosomenaberration auf. Von diesen 63 Feten waren bei 15,9 \% (10/63) sonographische Softmarker als alleinige Auffälligkeit diagnostiziert worden.

Die hier vorgestellten Ergebnisse weisen bereits auf eine deutliche Assoziation zwischen sonographischen Softmarken und fetalen Chromosomenanomalien hin. Inwieweit die einzelnen, von uns untersuchten Softmarker als diagnostisches Instrument zur Risikoabschätzung genutzt werden können, soll im Folgenden diskutiert werden. 


\subsection{Pyelektasie}

In dem von uns untersuchten, vorselektierten Gesamtkollektiv trat eine Pyelektasie bei insgesamt 192 Feten auf - in 169 Fällen (88,0 \%) lag sie isoliert vor, bei den übrigen Feten war sie mit weiteren Softmarkern assoziiert.

In der Literatur wird die Prävalenz der Pyelektasie in einem Normalkollektiv mit 0,72 bis 5,5\% angegeben. Tab. 22 zeigt die verschiedenen Häufigkeiten in den unterschiedlichen Studien:

\begin{tabular}{|c|c|c|c|}
\hline Autor & $\begin{array}{c}\text { Prävalenz der } \\
\text { Pyelektasie }\end{array}$ & Studienpopulation & cut off der Pyelektasie \\
\hline WiCKSTROM (1996) & $0,72 \%$ & 7481 & $\geq 4 \mathrm{~mm}(<33$. SSW) \\
\hline CHUDLEIGH et al. (2001) & $0,73 \%$ & 101600 & $\geq 5 \mathrm{~mm}$ \\
\hline BORRELLI et al. (2004) & $2,1 \%$ & 2900 & --- \\
\hline COCO und JEANTY (2005) & $2,9 \%$ & 12672 & --- \\
\hline LANGER et al. (1996) & $4,4 \%$ & 2170 & --- \\
\hline ISMAILI et al. (2003) & $4,5 \%$ & 5643 & $\geq 4 \mathrm{~mm}$ \\
\hline PERSUTTE et al. (1997) & $5,5 \%$ & 5529 & \\
\hline
\end{tabular}

Tab. 22: Prävalenz der Pyelektasie (modifiziert nach Coco und JEANTY (2005))

2,4 \% (4 / 169) der von uns untersuchten Feten mit einer isolierten Pyelektasie wiesen eine Chromosomenanomalie auf: alle vier Feten waren von einer Trisomie 21 betroffen.

Damit können wir uns der Aussage von ChudLEIGH et al. (2001) anschließen, die nach der Untersuchung von 737 Feten mit einer milden Pyelektasie $(5-10 \mathrm{~mm})$ vor allem das mit diesem Softmarker assoziierte erhöhte Risiko für eine Trisomie 21 beschreiben.

Es finden sich jedoch auch abweichende Beobachtungen: in dem Kollektiv von BORRELLI et al. (2004) wies keiner der Feten mit einer isolierten Pyelektasie eine Chromosomenaberration auf und bei COCO und JEANTY (2005) war es lediglich einer der 305 betroffenen Feten. Diese Ergebnisse können wir, wie oben bereits erläutert, nicht bestätigen.

Die von uns erhobenen Daten untermauern die bereits zuvor beschriebene Prädisposition männlicher Feten für eine Pyelektasie: innerhalb der Gruppe der 169 Feten mit einer isolierten Pyelektasie betrug das Verhältnis von männlichen zu weiblichen Feten 1,41:1. WAX et al. 
(2005) beschreiben hier sogar ein Verhältnis von 2,34:1 (für die isolierte Pyelektasie) und CoCo und JEANTY (2005) eines von 1,9:1 (für die nicht isoliert auftretende Pyelektasie).

Männliche Feten neigen aufgrund der besonderen anatomischen Beschaffenheit ihres Systems der ableitenden Harnwege zum Auftreten einer Pyelektasie. Daher weisen sie in der Regel eine höhere Prävalenz für diesen Softmarker auf.

Interessanterweise ist das Risiko der weiblichen, von einer isolierten Pyelektasie betroffenen Feten für eine Chromosomenaberration dahingegen höher als das Risiko der männlichen Feten. Chudleigh et al. (2001) fanden ein Anomalierisiko von 3,18\% (7 / 220) bei weiblichen, verglichen mit einem Risiko von nur 1,05 \% (5 / 474) bei männlichen Feten mit einem erweiterten Nierenbecken. Wurden die gonosomalen Anomalien außen vor gelassen, betrug das Risiko der weiblichen Feten nur noch 1,84\%.Wenngleich der Unterschied hier nicht signifikant war, so sprechen die Autoren dennoch von einem Trend für ein erhöhtes Anomalierisiko bei den weiblichen Feten.

Auch unsere Daten zeigen eine Tendenz zu der Annahme, dass sich das Risiko für eine Chromosomenaberration durch das Auftreten einer Pyelektasie bei weiblichen Feten stärker erhöht als bei männlichen Feten: in unserem Studienkollektiv wiesen 2,9\% (2/70) der weiblichen Feten mit einer isolierten Pyelektasie eine Chromosomenanomalie auf, verglichen mit nur 2,0 \% (2 / 99) der männlichen Feten. 


\subsection{Plexus-choroideus-Zysten (CPC)}

Plexus-choroideus-Zysten sind in einem Normalkollektiv in 0,6 bis $2,8 \%$ zu erwarten (siehe Tab. 23). Isolierte CPC finden sich bei 0,6 bis 2,4 \% der Feten (BROWN et al. 1999, MorCOS et al. 1998, GEARY et al. 1997, REINSCH 1997, WALKINSHAW et al. 1994).

\begin{tabular}{|c|c|c|c|}
\hline Autor & Prävalenz der CPC & Studienpopulation & cut off der CPC \\
\hline GEARY et al. (1997) & $0,6 \%$ & 13690 & $\geq 3 \mathrm{~mm}$ \\
\hline CHITTY et al. (1998) & $0,65 \%$ & 101600 & - -- \\
\hline GRAY et al. (1996) & $1,1 \%$ & 18861 & -- \\
\hline KUPFERMINC et al. (1994) & $1,1 \%$ & 9100 & $\geq 2 \mathrm{~mm}$ \\
\hline REINSCH (1997) & $1,87 \%$ & 16059 & 7617 \\
\hline MORCOS et al. (1998) & $2,8 \%$ & & $\geq$ \\
\hline
\end{tabular}

Tab. 23: Prävalenz der Plexus-choroideus-Zysten (modifiziert nach CHITTY et al. (1998))

Wir entdeckten Plexus-choroideus-Zysten bei insgesamt 45 Feten: 62,2 \% (28/45) wiesen bilaterale CPC auf, bei 11 Feten (24,4 \%) fanden sich Plexuszysten unilateral und bei den übrigen 6 Feten $(13,3 \%)$ wurde diesbezüglich keine Angabe gemacht.

33 dieser 45 Feten $(73,3 \%)$ wiesen Plexus-choroideus-Zysten als alleinige sonographische Auffälligkeit auf. Eine Chromosomenanomalie wurde bei 12,1 \% (4 / 33) dieser Feten mit isolierten CPC diagnostiziert: zwei Feten zeigten eine Trisomie 18 und zwei weitere wiesen eine strukturelle Anomalie (Addition) auf.

Die Tatsache, dass in unserem Kollektiv 6,1 \% (2 / 33) der Feten mit isolierten Plexuschoroideus-Zysten eine Trisomie 18 aufwiesen, passt zu der von NiCOLAIDES et al. (1986) erstmals beschriebenen Beobachtung eines Zusammenhangs zwischen diesem sonographischen Softmarker und dem EDWARDS-Syndrom. Diese Entdeckung konnte u. a. von CHITTY et al. (1998) bestätigt werden.

Alle vier der von einer Chromosomenanomalie betroffenen Feten in unserer Studienpopulation zeigten bilaterale Plexuszysten. Bezüglich der Lateralität der Plexuszysten und ihrer jeweiligen Assoziationsrate mit fetalen Chromosomenanomalien liegen unterschiedliche Aussagen vor: 
BROWN et al. (1999) sahen beispielsweise keine Assoziation zwischen unilateralen Plexuszysten und einem erhöhten Anomalierisiko. Dieser Meinung können wir uns anschließen, da Chromosomenanomalien in unserem Kollektiv ebenfalls nur bei Feten mit bilateralen Plexuszysten auftraten. Für unilaterale Plexuszysten konnten wir kein erhöhtes Anomalierisiko nachweisen. CHITTY et al. (1998) fanden dahingegen fetale Aneuploidien sowohl bei Feten mit uni- als auch bei solchen mit bilateralen Plexus-choroideus-Zysten.

Zusammenfassend sprechen unsere Daten auch bei diesem Softmarker für ein erhöhtes Fehlbildungsrisiko. Daher empfehlen wir, Schwangere im Falle der Entdeckung von isolierten, bilateralen CPC hinsichtlich der Durchführung einer Amniozentese zu beraten.

Dass in unserem Kollektiv der Feten mit einem positiven Softmarkerbefund außerdem alle Fälle von Trisomie 18 ausschließlich bei Feten mit CPC auftraten, deckt sich ebenfalls mit der bereits zuvor beschriebenen Erkenntnis, dass das Auftreten von Plexus-choroideus-Zysten insbesondere mit dieser speziellen Chromosomenanomalie assoziiert ist.

\subsection{Ventrikulomegalie, intrakardialer echogener Fokus (ICEF), singuläre Nabel- schnurarterie (SUA), echogener Darm und kurzes Femur / kurzer Humerus}

Aufgrund der in unserem Studienkollektiv sehr geringen Fallzahlen für die sonographischen Softmarker Ventrikulomegalie, ICEF, SUA, echogener Darm und kurzes Femur / kurzer Humerus soll auf diese fünf Marker im Folgenden nur kurz eingegangen werden.

In unserer speziellen Studienpopulation fand sich eine Ventrikulomegalie bei 15 Feten (isoliert bei 11 Feten). Von einer Chromosomenanomalie war nur einer der 11 Feten mit einer isolierten Ventrikulomegalie betroffen: er wies eine Triploidie auf. NICOLAIDES et al. (1990) beschreiben eine Anomalierate von $3 \%$ für ein Kollektiv von 35 Feten mit einer isolierten Ventrikulomegalie, woraus sie kein gesteigertes Anomalierisiko ableiten.

Intrakardiale echogene Foci (ICEF) entdeckten wir bei insgesamt 12 Feten. Dabei wies keiner der Feten mit isolierten ICEF $(n=6)$ eine Chromosomenanomalie auf. Diese Beobachtung unterstützt die Meinung von BRADLEY et al. (2005) und ANDERSON und JYOTI (2003), welche keine Assoziation zwischen dem Auftreten von isolierten ICEF und fetalen Chromosomenaberrationen sehen. WINTER et al. (2000) finden zwar eine Erhöhung des relativen Risikos für eine Trisomie $21 \mathrm{um}$ das 4,8fache bei Vorliegen von isolierten ICEF, allerdings konnten sie diese Risikosteigerung nur für ein Hochrisikokollektiv nachweisen.

Ähnlich verhält es sich mit unserem Ergebnis für die singuläre Nabelschnurarterie (SUA): hier wies nur ein einziger der von uns untersuchten Feten eine Chromosomenanomalie 
(Trisomie 21) auf, sodass für diesen Softmarker ebenfalls kein gesteigertes Anomalierisiko abgeleitet werden soll. GRANESE et al. (2007), LUBUSKY et al. (2007) sowie ChrisTINA et al. (2005) fanden eine erhöhte Anomalierate nur bei Feten, die außer der SUA noch weitere Malformationen aufwiesen. Für die isolierte SUA konnten sie keine Zunahme bei der Assoziationsrate nachweisen. BUDORICK et al. (2001) konnten ebenfalls kein erhöhtes Anomalierisiko für die isolierte SUA nachweisen, allerdings fanden sie ein signifikante Risikosteigerung für das Auftreten einer kardialen Malformation.

Beim echogenen Darm und dem kurzen Femur / kurzen Humerus bieten unsere geringen Gesamtfallzahlen von nur einem bzw. vier Feten keinen Spielraum für Interpretationen.

Einige Autoren beschreiben eine Assoziation zwischen dem Auftreten eines echogenen Darms und fetalen Chromosomenanomalien. So sahen Al-KouAtly et al. (2001), welche insgesamt 175 Feten mit echogenem Darm (isoliert und nicht-isoliert) untersuchten, ein erhöhtes Risiko sowohl für eine Aneuploidie als auch für die Zystische Fibrose (CF) und eine Infektion mit dem Zytomegalievirus (CMV). Auch SIMON-BOUY et al. (2003), BERLIN et al. (1999) sowie MulLeR et al. (1995) empfehlen eine Karyotypisierung des Feten, wenn im pränatalen Ultraschall ein echogener Darm entdeckt wird.

Für die sonographischen Softmarker kurzes Femur bzw. kurzer Humerus beschreiben NyBERG et al. (1993 b) eine Risikoerhöhung für eine Trisomie 21 um das 11 fache, wenn ein Fet sowohl ein kurzes Femur als auch einen kurzen Humerus aufweist.

\subsection{Fetale Fehlbildungen und andere Marker}

Im Folgenden soll auf die Gruppe der Feten mit Fehlbildungen und anderen Markern eingegangen werden. Diese standen zwar nicht direkt im Fokus der vorliegenden Arbeit, dienten der Gruppe der Feten mit positivem Softmarkerbefund aber als Vergleichskollektiv (siehe Kapitel 3.1.2, S. 21).

Unter den Feten mit Fehlbildungen und anderen Markern lag das mütterliche Alter im Durchschnitt bei 28,5 Jahren, und die mittlere Schwangerschaftsdauer betrug zum Zeitpunkt der Amniozentese 23,5 SSW.

Mit 28,5 \% (59 / 207) waren die am häufigsten vertretenen fetalen Fehlbildungen die Fehlbildungen des Zentralen Nervensystems (ZNS). Von den so genannten „anderen Markern“ traten die Abweichungen in der Fruchtwassermenge (Poly-, Oligo- und Anhydramnion) mit 48,3 \% (84 / 174) am häufigsten auf. 
Im Kollektiv der Feten mit Fehlbildungen und anderen Markern lag die Assoziationsrate der entdeckten Auffälligkeiten im pränatalen Ultraschall mit fetalen Chromosomenanomalien erwartungsgemäß signifikant höher (15,2\%), als in der Gruppe der Feten mit einem positiven Softmarkerbefund (3,9\%, siehe Kapitel 9.2.4, S. 60).

Unter den Chromosomenanomalien war die Trisomie 18 (EDWARDS-Syndrom) mit 27,5 \% (14 / 51) diejenige Anomalie, welche unter den Feten mit Fehlbildungen und anderen Markern am häufigsten anzutreffen war. Hierbei unterscheiden sich die Hardmarker also von den Softmarkern, bei welchen die Trisomie 21 die häufigste Chromosomenanomalie darstellte.

Der Grund hierfür liegt in ihrer Eigenschaft als so genannte ,echte“ Fehlbildungen begründet: als Hardmarker sind die fetalen Fehlbildungen gerade nicht nur ein Indikator für eine möglicherweise bestehende Chromosomenanomalie des Feten, sondern in einigen Fällen sind sie beinahe schon beweisend für das Vorliegen eines bestimmten kindlichen Fehlbildungssyndroms.

Den Trisomien 18 und 13 ist gemeinsam, dass sie mit schweren anatomischen Fehlbildungen des Feten einhergehen. So kommt es bei diesen Chromosomenanomalien unter anderem zu einer Mikrozephalie mit Fehlbildungen des Zentralen Nervensystems (ZNS), zu einem Hygrom, einer Polydaktylie, zu Zwerchfellhernien und zu einem Polyhydramnion.

Da diese Fehlbildungen meist zu einer erheblichen Beeinträchtigung der Funktion des betroffenen Organsystems führen, kommt es bei der Trisomie 18 (EDWARDS-Syndrom) in ca. $95 \%$, und bei der Trisomie 13 (PÄTAU-Syndrom) sogar in ca. $98 \%$ der Fälle zu einem spontanen Abort (SNIJDERS et al. 1994).

Anders verhält es sich dahingegen bei der Trisomie 21: die von einem Down-Syndrom betroffenen Feten weisen in der Regel lediglich Softmarker auf, welche im Gegensatz zu den Hardmarkern eben nicht zu einer Funktionseinschränkung des fetalen Organismus führen. Daher liegt die Abortrate bei dieser Chromsomenanomalie mit 70 bis $80 \%$ etwas niedriger (SNIJDERS et al. 1994).

Diese Zahlen deuten zwar einerseits darauf hin, dass die Softmarker insgesamt einen benigneren Charakter haben als die Hardmarker, andererseits zeigen sie aber gleichzeitig, dass auch beim Vorliegen von Softmarkern stets mit einem erhöhten Risiko für eine fetale Chromosomenanomalie gerechnet werden muss. 


\subsection{Bedeutung der sonographischen Softmarker für das pränatale Screening}

In den Mutterschafts-Richtlinien sind für den Verlauf der Schwangerschaft insgesamt drei Ultraschalluntersuchungen vorgesehen: eine in der Frühschwangerschaft (9. bis 12. SSW), eine im II. Trimenon (19. bis 22. SSW) und eine im III. Trimenon (29. bis 32. SSW) (siehe Kapitel 1.2, S. 6).

Diese Screening-Untersuchungen dienen der Überwachung einer normal verlaufenden Schwangerschaft. Sie beinhalten einerseits die Feststellung der intrauterinen Lage des Embryos, den Nachweis bzw. Ausschluss einer Mehrlingsschwangerschaft und die Bestimmung der Plazentalokalisation.

Andererseits sehen die Richtlinien - insbesondere im II. und III. Trimenon - die Bestimmung verschiedener biometrischer Daten des Feten (biparietaler Durchmesser (BPD) etc.) vor. Diese Maße dienen u. a. der Abschätzung des Gestationsalters.

Wenn sich im Rahmen dieser Screening-Untersuchungen fetale Auffälligkeiten zeigen, welche nach Einschätzung des behandelnden Gynäkologen eine weitere Abklärung erforderlich machen, wird die Schwangere zur Durchführung einer gezielten Ultraschalldiagnostik weiter überwiesen. Diese ist dann zwar ebenfalls noch Bestandteil der Mutterschaftsvorsorge, gehört aber nicht mehr zum Screening.

Eine solche gezielte Ultraschalldiagnostik wird v. a. von älteren Schwangeren häufig als Alternative $\mathrm{zu}$ den verschiedenen invasiven diagnostischen Methoden in Anspruch genommen, um eine fetale Chromosomenanomalie auszuschließen. Dabei ist es von größter Bedeutung, die werdenden Eltern darüber aufzuklären, dass das Risiko für das Vorliegen einer Chromosomenanomalie beim Feten im Falle des Fehlens charakteristischer sonographischer Befunde lediglich gemindert, aber keinesfalls vollständig ausgeschlossen werden kann (Arbeitsgemeinschaft der Wissenschaftlichen Medizinischen Fachgesellschaften 2008).

Einer der oben genannten biometrischen Parameter, der laut Mutterschafts-Richtlinien im Rahmen des Ultraschall-Screenings erfasst wird, ist die Längenmessung des Femur bzw. Humerus. Hierbei muss betont werden, dass die Bestimmung der Länge dieser beiden Röhrenknochen hier nicht in ihrer Eigenschaft als Softmarker und damit auch nicht als diagnostisches Kriterium für die Detektion fetaler Chromosomenanomalien fungiert. Vielmehr dient sie im II. und III. Trimenon der Überprüfung einer zeitgerechten Entwicklung des Feten. 
Auch die übrigen Softmarker zählen zu den Parametern der ergänzenden sonographischen Feindiagnostik, und sind damit nicht mehr Bestandteil des Screenings.

Die Ergebnisse der vorgelegten Arbeit berechtigen jedoch zu der Forderung, über die Aufnahme einzelner sonographischer Softmarker in das pränatale Ultraschall-Screening zu diskutieren, um so allen Schwangeren diese effektive, komplikationsarme und kostengünstige Methode zur genaueren Risikoabschätzung einer fetalen Chromosomenanomalie ermöglichen zu können.

Nach unserer Datenlage sollte dies vor allem für die Pyelektasie und die Plexus-choroideusZysten (CPC) überlegt werden. Diese beiden Softmarker scheinen mit Anomalieraten von 2,4 bzw. 12,1 \% eine suffiziente Aussagekraft zu besitzen, um als Screeningparameter zur Detektion fetaler Chromosomenanomalien in Erwägung gezogen zu werden. 


\section{Zusammenfassung}

Im Mittelpunkt der vorliegenden Arbeit stehen die sonographischen Softmarker. Als Softmarker bezeichnet man geringgradige, primär unspezifische Strukturauffälligkeiten in der Anatomie des Feten, welche mittels pränatalen Ultraschalls diagnostiziert werden können. In der Regel führt das Vorliegen von Softmarkern nicht zu einer Beeinträchtigung der Funktion des betroffenen fetalen Organsystems.

Die sonographischen Softmarker haben sich im Verlauf der letzten Jahre zu einem wichtigen Bestandteil der pränatalen Diagnostik entwickelt, da sie zu einem gewissen Prozentsatz mit dem Auftreten von fetalen Chromosomenanomalien assoziiert sind. Diese Eigenschaft macht sie zu einem wertvollen diagnostischen Parameter, welcher für die Beratung der werdenden Eltern hinsichtlich eines erhöhten fetalen Anomalierisikos genutzt werden kann.

In dem Bestreben, einen Beitrag zu der teils sehr kontroversen Diskussion liefern zu können, ob und wann der Entdeckung sonographischer Softmarker weitere und ggf. auch invasive Untersuchungen zur Fehlbildungsdiagnostik folgen sollten, wurden die über einen Zeitraum von zehn Jahren erhobenen Daten von insgesamt 594 Schwangeren retrospektiv evaluiert.

Bei 259 dieser Patientinnen wurde die Indikation zur Amniozentese mit dem Ziel der fetalen Chromosomenanalyse ausschließlich aufgrund von sonographischen Softmarkern gestellt, d. h. die betroffenen Feten wiesen keine zusätzlichen Auffälligkeiten auf.

Es zeigte sich, dass das Vorhandensein von sonographischen Softmarkern mit einer signifikanten Risikoerhöhung für fetale Chromosomenanomalien einhergeht. Mit einer Anomalierate von 3,9 \% (10 / 259) lag die Häufigkeit fetaler Chromosomenaberrationen in unserem Kollektiv der Feten mit positivem Softmarkerbefund rund 16mal höher, als das altersabhängige Hintergrundrisiko vermuten ließe.

Unsere Ergebnisse sprechen dafür, dass eine isoliert auftretende Pyelektasie mit einer signifikanten Erhöhung des Risikos insbesondere für die Trisomie 21 (Down-Syndrom) assoziiert ist. 2,4 \% (4/169) der Feten mit einer isolierten Pyelektasie wiesen eine Chromosomenanomalie auf, wobei alle vier Feten von einer Trisomie 21 betroffen waren.

Zudem zeigen unsere Daten eine Tendenz zu der bereits von anderen Autoren beschriebenen Beobachtung, dass das Auftreten einer Pyelektasie bei weiblichen Feten mit einer stärkeren Erhöhung des Anomalierisikos einhergeht, als dies bei männlichen Feten der Fall ist, obwohl männliche Feten insgesamt eine Prädisposition für diesen Softmarker aufweisen.

Des Weiteren zeigten 12,1 \% (4/33) der Feten mit isolierten, bilateralen Plexus-choroideusZysten (CPC) eine Chromosomenanomalie, was ebenfalls eine signifikante Erhöhung des 
Anomalierisikos bedeutet. Dass zwei dieser vier Feten von einer Trisomie 18 (EDWARDSSyndrom) betroffen waren, zeigt die bereits in früheren Arbeiten beschriebene, deutliche Assoziation der CPC mit eben dieser Anomalie.

Auf der Grundlage der durch diese Studie gewonnenen Erkenntnisse empfehlen wir daher, die werdenden Eltern über das erhöhte Risiko für eine fetale Chromosomenaberration aufzuklären, welches mit dem Auftreten einer isolierten Pyelektasie und isolierten, bilateralen Plexus-choroideus-Zysten einhergeht.

Sollte ein Fet von einer Pyelektasie oder von bilateralen Plexus-choroideus-Zysten betroffen sein, können der Schwangeren zwei unterschiedliche Möglichkeiten zur weiteren Fehlbildungsdiagnostik angeboten werden: Einerseits kann zum definitiven Ausschluss einer fetalen Chromosomenanomalie eine Amniozentese vorgenommen werden, die - als invasive Maßnahme - mit einem entsprechenden Risiko behaftet ist. Andererseits kann durch serielle Ultraschall-Untersuchungen ohne pathologischen Befund zumindest eine Risikoreduktion für eine fetale Chromosomenaberration konstatiert werden. 


\section{Verzeichnis der Tabellen}

Tab. 1: $\quad$ Organsysteme, die im Rahmen der sonographischen Feindiagnostik untersucht werden

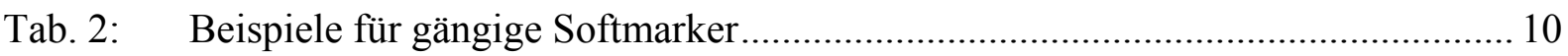

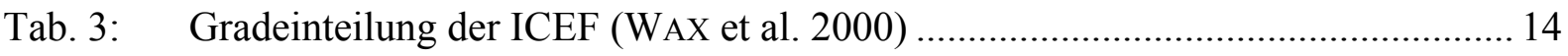

Tab. 4: Ultraschallanomalien, die für die vorliegende Studie untersucht wurden .............22

Tab. 5: Unterschiede bzgl. des mittleren mütterlichen Alters und der mittleren

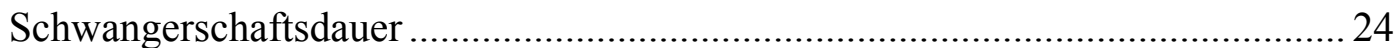

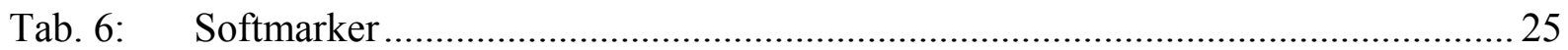

Tab. 7: Verteilung der einzelnen Softmarker ................................................................ 26

Tab. 8: Kombinationen von Softmarkern .................................................................... 27

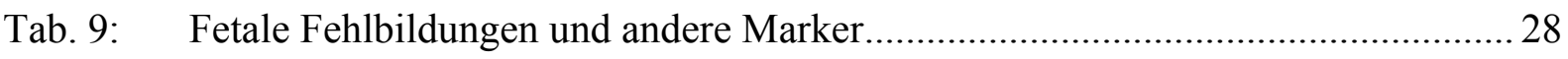

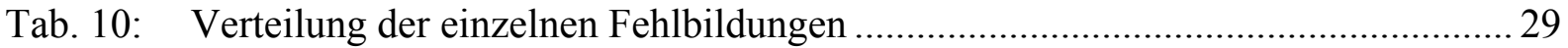

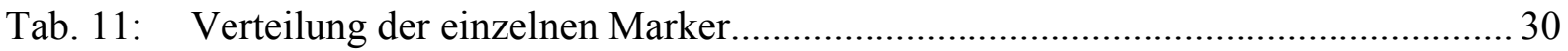

Tab. 12: Kombinationen von Fehlbildungen untereinander .............................................. 31

Tab. 13: Kombinationen von Fehlbildungen und anderen Markern ................................... 31

Tab. 14. Kombinationen der anderen Marker untereinander .............................................. 32

Tab. 15: Verteilung der Chromosomenanomalien im Gesamtkollektiv .............................. 33

Tab. 16: Verteilung der Chromosomenanomalien bei Patientinnen mit positivem

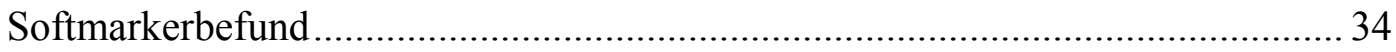

Tab. 17: Feten mit einer Chromosomenanomalie und positivem Softmarkerbefund........... 35

Tab. 18: Verteilung der Chromosomenanomalien in der Gruppe ,fetale Fehlbildungen und andere Marker“

Tab. 19: Häufigkeit von Chromosomenanomalien in Abhängigkeit vom mütterlichen Alter

Tab. 20: Häufigkeit von Chromosomenanomalien bei Feten mit einer Pyelektasie ............ 39

Tab. 21: Häufigkeit von Chromosomenanomalien bei Feten mit CPC ............................... 40

Tab. 22: Prävalenz der Pyelektasie (modifiziert nach CocO und JEANTY (2005)) .............. 47

Tab. 23: Prävalenz der Plexus-choroideus-Zysten (modifiziert nach

CHITTY et al. (1998)) 


\section{Verzeichnis der Abbildungen}

Abb. 1: Bilaterale renale Pyelektasie (a. p.) (VAN DEN Hof et al. 2005) ............................ 11

Abb. 2: $\quad$ Plexus-choroideus-Zyste (VAN DEN HoF et al. 2005) ........................................... 12

Abb. 3: Leicht erweitertes Hinterhorn des Seitenventrikels (VAN DEN Hof et al. 2005) ... 13

Abb. 4: Echogener intrakardialer Fokus im linken Ventrikel (VAN DEN HoF et al. 2005) . 14

Abb. 5: $\quad$ SUA (im Querschnitt der Nabelschnur) (VAN DEN Hof et al. 2005)..................... 15

Abb. 6: Fetaler echogener Darm (VAN DEN Hof et al. 2005) ........................................... 16

Abb. 7: Erhöhte Nackentransparenz (The Fetal Medicine Foundation 2009) .................... 17

Abb. 8: Unterschiede in der Verteilung des mütterlichen Alters ...................................... 24

Abb. 9: Unterschiede in der Verteilung der Chromosomenanomalien .............................. 37 


\section{Anhang}

\subsection{Standardabweichung}

$s=\sqrt{\frac{1}{n-1} \sum_{v=1}^{n}\left(x_{v}-\bar{x}\right)^{2}}$

\subsection{Vergleich zweier Wahrscheinlichkeiten}

9.2.1 Wahrscheinlichkeit von Chromosomenanomalien im Falle des Vorliegens einer Pyelektasie, abhängig vom fetalen Geschlecht

$\mathrm{p}_{1}$ : Wahrscheinlichkeit für eine Anomalie bei weiblichen Feten

$\mathrm{p}_{2}$ : Wahrscheinlichkeit für eine Anomalie bei männlichen Feten

$\mathrm{H}: \quad \mathrm{p}_{1} \leq \mathrm{p}_{2}$

$\mathrm{G}: \quad \mathrm{p}_{1}>\mathrm{p}_{2}$

$\hat{p}=\frac{70 \cdot 0,043+99 \cdot 0,02}{70+99}=0,03$

$T=\frac{0,043-0,02}{\sqrt{0,03(1-0,03)\left(\frac{1}{70}+\frac{1}{99}\right)}}=0,863$

Wegen $0,863<\mathrm{z}_{0,95}=1,695$ ist das Ergebnis nicht signifikant zum Niveau 0,005.

\subsubsection{Wahrscheinlichkeit von Chromosomenanomalien bei positivem Softmarker-} befund, abhängig vom mütterlichen Alter

$\mathrm{p}_{1}$ : Wahrscheinlichkeit für eine Anomalie bei Schwangeren $\geq 35$ Jahre

$\mathrm{p}_{2}: \quad$ Wahrscheinlichkeit für eine Anomalie bei Schwangeren $<35$ Jahre

$\mathrm{H}: \quad \mathrm{p}_{1} \leq \mathrm{p}_{2}$

$\mathrm{G}: \quad \mathrm{p}_{1}>\mathrm{p}_{2}$

$\hat{p}=\frac{41 \cdot 0,049+217 \cdot 0,037}{41+217}=0,038$

$T=\frac{0,049-0,037}{\sqrt{0,038(1-0,038)\left(\frac{1}{41}+\frac{1}{217}\right)}}=0,369$

Wegen $0,369<z_{0,95}=1,695$ ist das Ergebnis nicht signifikant zum Niveau 0,005. 
9.2.3 Wahrscheinlichkeit einer Trisomie 18 bei Vorliegen von isolierten CPC (verglichen mit einem Kollektiv von GRAY et al. (1996))

$\mathrm{p}_{1}$ : Wahrscheinlichkeit einer Trisomie 18 bei CPC (unser Studienkollektiv)

$\mathrm{p}_{2}$ : Wahrscheinlichkeit einer Trisomie 18 bei CPC (GRAY et al. (1996))

$\mathrm{H}: \quad \mathrm{p}_{1} \leq \mathrm{p}_{2}$

$\mathrm{G}: \quad \mathrm{p}_{1}>\mathrm{p}_{2}$

$\hat{p}=\frac{33 \cdot 0,061+208 \cdot 0,034}{33+208}=0,037$

$T=\frac{0,061-0,034}{\sqrt{0,037(1-0,037)\left(\frac{1}{33}+\frac{1}{208}\right)}}=0,759$

Wegen $0,759<\mathrm{z}_{0,95}=1,695$ ist das Ergebnis nicht signifikant zum Niveau 0,005.

9.2.4 Unterschiede in der Wahrscheinlichkeit von Chromosomenanomalien in den unterschiedlichen Vergleichsgruppen

$\mathrm{p}_{1}$ : Wahrscheinlichkeit für eine Anomalie bei Fehlbildungen und / oder anderen Markern

$\mathrm{p}_{2}$ : Wahrscheinlichkeit für eine Anomalie bei positivem Softmarkerbefund

$\mathrm{H}: \quad \mathrm{p}_{1} \leq \mathrm{p}_{2}$

$\mathrm{G}: \quad \mathrm{p}_{1}>\mathrm{p}_{2}$

$\hat{p}=\frac{335 \cdot 0,152+259 \cdot 0,039}{335+259}=0,103$

$T=\frac{0,152-0,039}{\sqrt{0,103(1-0,103)\left(\frac{1}{335}+\frac{1}{259}\right)}}=4,493$

Wegen 4,493 $>z_{0,95}=1,695$ ist das Ergebnis signifikant zum Niveau 0,005. 


\section{Literaturverzeichnis}

Abuhamad AZ, Shaffer W, Mari G, Copel JA, Hobbins JC, Evans AT (1995): Single umbilical artery: does it matter which artery is missing? Am J Obstet Gynecol 173, $728-732$

Ahmad G, Green P (2005): Outcome of fetal pyelectasis diagnosed antenatally. J Obstet Gynaecol 25, 119-122

Al-Kouatly HB, Chasen ST, Streltzoff J, Chervenak FA (2001): The clinical significance of fetal echogenic bowel. Am J Obstet Gynecol 185, 1035-1038

Anderson N, Jyoti R (2003): Relationship of isolated fetal intracardiac echogenic focus to trisomy 21 at the mid-trimester sonogram in women younger than 35 years. Ultrasound Obstet Gynecol 21, 354-358

Arbeitsgemeinschaft der Wissenschaftlichen Medizinischen Fachgesellschaften (AWMF): Ultraschalldiagnostik im Rahmen der Schwangerenvorsorge, aus: AWMF online, 2008. URL: http://www.uni-duesseldorf.de/AWMF/11/015-044.htm (Stand: 12.12.2009)

Batukan C, Holzgreve W, Visca E, Tercanli S (2001): [Ultrasound indications of fetal chromosome abnormalities in the 2nd trimester]. Praxis (Bern 1994) 90, 786-795

Berlin BM, Norton ME, Sugarman EA, Tsipis JE, Allitto BA (1999): Cystic fibrosis and chromosome abnormalities associated with echogenic fetal bowel. Obstet Gynecol $\underline{94}$, $135-138$

Bettelheim D, Deutinger J, Bernaschek G (1999): The value of echogenic foci ('golfballs') in the fetal heart as a marker of chromosomal abnormalities. Ultrasound Obstet Gynecol $\underline{14}$, 98-100 
Bettelheim D, Kolinek B, Schaller A, Bernaschek G (2002): Complication rates of invasive intrauterine procedures in a centre for prenatal diagnosis and therapy. Ultraschall Med $\underline{23}, 119-122$

Borrelli AL, Borrelli P, Di Domenico A, Felicetti M, Corcione M, Torella M (2004): The incidence of chromosomal anomalies in fetuses affected by mild renal pyelectasis. Minerva Ginecol $\underline{56}, 137-140$

Borruto F, Comparetto C, Acanfora L, Bertini G, Rubaltelli FF (2002): Role of ultrasound evaluation of nuchal translucency in prenatal diagnosis. Clin Exp Obstet Gynecol 29, $235-241$

Bradley KE, Santulli TS, Gregory KD, Herbert W, Carlson DE, Platt LD (2005): An isolated intracardiac echogenic focus as a marker for aneuploidy. Am J Obstet Gynecol 192, 2021-2026

Bromley B, Lieberman E, Shipp TD, Benacerraf BR (2002): The genetic sonogram: a method of risk assessment for Down syndrome in the second trimester. J Ultrasound Med $\underline{21}$, 1087-1096

Brown T, Kliewer MA, Hertzberg BS, Ruiz C, Stamper TH, Rosnes J, Lucas A, Wright LN, Chescheir NC, Farmer L (1999): A role for maternal serum screening in detecting chromosomal abnormalities in fetuses with isolated choroid plexus cysts: a prospective multicentre study. Prenat Diagn $\underline{19}, 405-410$

Budorick NE, Kelly TF, Dunn JA, Scioscia AL (2001): The single umbilical artery in a highrisk patient population: what should be offered? J Ultrasound Med 20, 619-627

Cardoza JD, Goldstein RB, Filly RA (1988): Exclusion of fetal ventriculomegaly with a single measurement: the width of the lateral ventricular atrium. Radiology $\underline{169}, 711-714$ 
Chitty LS, Chudleigh P, Wright E, Campbell S, Pembrey M (1998): The significance of choroid plexus cysts in an unselected population: results of a multicenter study. Ultrasound Obstet Gynecol 12, 391-397

Christina MP, Ana G, Inés T, Manuel GE, Enrique IG (2005): Perinatal results following the prenatal ultrasound diagnosis of single umbilical artery. Acta Obstet Gynecol Scand $\underline{84}$, 1068-1074

Chudleigh PM, Chitty LS, Pembrey M, Campbell S (2001): The association of aneuploidy and mild fetal pyelectasis in an unselected population: the results of a multicenter study. Ultrasound Obstet Gynecol 17, 197-202

Coco C, Jeanty P (2005): Isolated fetal pyelectasis and chromosomal abnormalities. Am J Obstet Gynecol 193, 732-738

Cohen-Overbeek TE, Wijngaard-Boom P, Ursem NT, Hop WC, Wladimiroff JW, Wolffenbuttel KP (2005): Mild renal pyelectasis in the second trimester: determination of cut-off levels for postnatal referral. Ultrasound Obstet Gynecol 25, 378-383

Damen-Elias HA, Luijnenburg SE, Visser GH, Stoutenbeek PH, de Jong TP (2005): Mild pyelectasis diagnosed by prenatal ultrasound is not a predictor of urinary tract morbidity in childhood. Prenat Diagn 25, 1239-1247

Dicke JM, Crane JP (1992): Sonographically detected hyperechoic fetal bowel: significance and implications for pregnancy management. Obstet Gynecol 무, 778-782

Dirks K: Patienteninformation Ultraschall - Sonographie, aus: Deutsche Gesellschaft für Ultraschall in der Medizin (DEGUM), 2004. URL: http://www.degum.de/163.html (Stand: 15.05.2009)

Eiben B, Glaubitz R (2005): First-trimester screening: an overview. J Histochem Cytochem $\underline{53}, 281-283$ 
Felberbaum RE, Diedrich K, Jonat W, Ortmann O, Crohns C, Hußlein EM: Facharztprüfung Gynäkologie und Geburtshilfe in Fällen, Fragen und Antworten. 1. Auflage; Elsevier GmbH, München 2005

Felicetti M, Domenico AD, Salzano P, Borrelli P, Laboccetta A, Borrelli AL (2001): The importance of high resolution transvaginal sonography in early screening of fetal chromosomal pathology. Clin Exp Obstet Gynecol 28, 53-54

Filly RA, Cardoza JD, Goldstein RB, Barkovich AJ (1989): Detection of fetal central nervous system anomalies: a practical level of effort for a routine sonogram. Radiology 172 , $403-408$

Font GE, Solari M (1998): Prenatal diagnosis of bowel obstruction initially manifested as isolated hyperechoic bowel. J Ultrasound Med 17, 721-723

Friese K: Sprechstunde pränataler Ultraschall, aus: Ludwig-Maximilians-Universität (LMU), o. J. URL: http://gyngh.klinikum.uni-muenchen.de/sprechstunden/ss_ultraprae.html (Stand: 15.05.2009)

Geary M, Patel S, Lamont R (1997): Isolated choroid plexus cysts and association with fetal aneuploidy in an unselected population. Ultrasound Obstet Gynecol 10, 171-173

Geipel A, Germer U, Welp T, Schwinger E, Gembruch U (2000): Prenatal diagnosis of single umbilical artery: determination of the absent side, associated anomalies, Doppler findings and perinatal outcome. Ultrasound Obstet Gynecol 15, 114-117

Gemeinsamer Bundesausschuss (2009): Richtlinien des Bundesausschusses der Ärzte und Krankenkassen über die ärztliche Betreuung während der Schwangerschaft und nach der Entbindung (,Mutterschafts-Richtlinien“), Bundesanzeiger $\underline{40,} 946$

Ghose I, Mason GC, Martinez D, Harrison KL, Evans JA, Ferriman EL, Stringer MD (2000): Hyperechogenic fetal bowel: a prospective analysis of sixty consecutive cases. BJOG 107, 426-429 
Granese R, Coco C, Jeanty P (2007): The value of single umbilical artery in the prediction of fetal aneuploidy: findings in 12,672 pregnant women. Ultrasound Q 23, 117-121

Gray DL, Winborn RC, Suessen TL, Crane JP (1996): Is genetic amniocentesis warranted when isolated choroid plexus cysts are found? Prenat Diagn $\underline{16}$, 983-990

Hormon Zentrum München: Die Indikation, aus: Hormon Zentrum München, 2009. URL: http://www.hormonzentrum.de/genetik/praenataldiagnostik/die-indikation.html (Stand: 23.05.2009)

Ismaili K, Hall M, Donner C, Thomas D, Vermeylen D, Avni FE (2003): Results of systematic screening for minor degrees of fetal renal pelvis dilatation in an unselected population. Am J Obstet Gynecol 188, 242-246

Jackson M, Rose NC (1998): Diagnosis and management of fetal nuchal translucency. Semin Roentgenol 33, 333-338

Jemmali M, Valat AS, Poulain P, Favre R, Bourgeot P, Subtil D, Puech F (1999): [Nuchal translucency: screening for chromosomal abnormalities and congenital malformations. Multicenter study]. J Gynecol Obstet Biol Reprod (Paris) 28

Kupferminc MJ, Tamura RK, Sabbagha RE, Parilla BV, Cohen LS, Pergament E (1994): Isolated choroid plexus cyst(s): an indication for amniocentesis. Am J Obstet Gynecol $\underline{171}, 1068-1071$

Kurjak A, Kos M, Stipoljev F, Latin V, Funduk-Kurjak B, Kos M, Miskovic B (1999): Ultrasonic markers of fetal chromosomal abnormalities. Eur J Obstet Gynecol Reprod Biol $\underline{85}, 105-108$

Langer B, Simeoni U, Montoya Y, Casanova R, Schlaeder G (1996): Antenatal diagnosis of upper urinary tract dilation by ultrasonography. Fetal Diagn Ther $\underline{11}$, 191-198 
Lubusky M, Dhaifalah I, Prochazka M, Hyjanek J, Michova I, Vomackova K, Santavy J (2007): Single umbilical artery and its siding in the second trimester of pregnancy: relation to chromosomal defect. Prenat Diagn $\underline{27}$, 327-331

Malone FD, Canick JA, Ball RH, Nyberg DA, Comstock CH, Bukowski R, Berkowitz RL, Gross SJ, Dugoff L, Craigo SD (2005): First-trimester or second-trimester screening, or both, for Down's syndrome. N Engl J Med $\underline{353}$, 2001-2011

Morcos CL, Platt LD, Carlson DE, Gregory KD, Greene NH, Korst LM (1998): The isolated choroid plexus cyst. Obstet Gynecol 92, 232-236

Mujezinovic F, Alfirevic Z (2007): Procedure-related complications of amniocentesis and chorionic villous sampling: a systematic review. Obstet Gynecol 110, 687-694

Muller F, Dommergues M, Aubry MC, Simon-Bouy B, Gautier E, Oury JF, Narcy F (1995): Hyperechogenic fetal bowel: an ultrasonographic marker for adverse fetal and neonatal outcome. Am J Obstet Gynecol 173, 508-513

Nicolaides KH (2005): First-trimester screening for chromosomal abnormalities. Semin Perinatol 29, 190-194

Nicolaides KH, Azar G, Byrne D, Mansur C, Marks K (1992): Fetal nuchal translucency: ultrasound screening for chromosomal defects in the first trimester of pregnancy. BMJ $\underline{304}, 867-869$

Nicolaides KH, Berry S, Snijders RJ, Thorpe-Beeston JG, Gosden C (1990): Fetal lateral cerebral ventriculomegaly: associated malformations and chromosomal defects. Fetal Diagn Ther $\underline{5}, 5-14$

Nicolaides KH, Gosden CM, Rodeck CH (1986): Rapid karyotyping in non-lethal fetal malformations. Lancet $\underline{\mathrm{i}}, 283-287$ 
Nyberg DA, Dubinsky T, Resta RG, Mahony BS, Hickok DE, Luthy DA (1993 a): Echogenic fetal bowel during the second trimester: clinical importance. Radiology $\underline{188}, 527-531$

Nyberg DA, Resta RG, Luthy DA, Hickok DE, Williams MA (1993 b): Humerus and femur length shortening in the detection of Down's syndrome. Am J Obstet Gynecol $\underline{168}$, $534-538$

Parra-Cordero M, Quiroz L, Rencoret G, Pedraza D, Muñoz H, Soto-Chacón E, MirandaMendoza I (2007): Screening for trisomy 21 during the routine second-trimester ultrasound examination in an unselected Chilean population. Ultrasound Obstet Gynecol $\underline{30}, 946-951$

Pauer HU, Viereck V, Krauss V, Osmers R, Krauss T (2003): Incidence of fetal malformations in pregnancies complicated by oligo- and polyhydramnios. Arch Gynecol Obstet $\underline{268}, 52-56$

Persutte WH, Koyle M, Lenke RR, Klas J, Ryan C, Hobbins JC (1997): Mild pyelectasis ascertained with prenatal ultrasonography is pediatrically significant. Ultrasound Obstet Gynecol 10, 12-18

Petrikovsky BM, Challenger M, Wyse LJ (1995): Natural history of echogenic foci within ventricles of the fetal heart. Ultrasound Obstet Gynecol $\underline{5}, 92-94$

Pilu G, Falco P, Gabrielli S, Perolo A, Sandri F, Bovicelli L (1999): The clinical significance of fetal isolated cerebral borderline ventriculomegaly: report of 31 cases and review of the literature. Ultrasound Obstet Gynecol 14, 320-326

Raniga S, Desai PD, Parikh H (2006): Ultrasonographic soft markers of aneuploidy in second trimester: are we lost? MedGenMed $\underline{8}, 9$

Reinsch RC (1997): Choroid plexus cysts - association with trisomy: prospective review of 16,059 patients. Am J Obstet Gynecol 176, 1381-1383 
Sancken U (2000): Der so genannte Triple-Test. Dtsch Arztebl 97, 532-537

Senat MV, Bernard JP, Schwärzler P, Britten J, Ville Y (1999): Prenatal diagnosis and follow-up of 14 cases of unilateral ventriculomegaly. Ultrasound Obstet Gynecol 14, $327-332$

Sepulveda W, Reid R, Nicolaidis P, Prendiville O, Chapman RS, Fisk NM (1996): Secondtrimester echogenic bowel and intraamniotic bleeding: association between fetal bowel echogenity and amniotic fluid spectrophotometry at $410 \mathrm{~nm}$. Am J Obstet Gynecol 174, $839-842$

Simon-Bouy B, Muller F; Groupe Collaboratif Français (2003): [Hyperechogenic fetal bowel: collaborative study of 682 cases]. J Gynecol Obstet Biol Reprod (Paris) 32, 459-465

Snijders RJ, Holzgreve W, Cuckle H, Nicolaides KH (1994): Maternal age-specific risks for trisomies at 9-14 weeks' gestation. Prenat Diagn 14, 543-552

Snijders RJ, Sundberg K, Holzgreve W, Henry G, Nicolaides KH (1999): Maternal age- and gestation-specific risk for trisomy 21. Ultrasound Obstet Gynecol 13, 167-170

Sohl BD, Scioscia AL, Budorick NE, Moore TR (1999): Utility of minor ultrasonographic markers in the prediction of abnormal fetal karyotype at a prenatal diagnostic center. Am J Obstet Gynecol 181, 898-903

Tannirandorn Y, Manotaya S, Uerpairojkit B, Tanawattanacharoen S, Wacharaprechanont T, Charoenvidhya D (2002): Value of humerus length shortening for prenatal detection of Down syndrome in a Thai population. J Obstet Gynaecol Res $\underline{28}, 89-94$

Tercanli S, Holzgreve W, Batukan C, Gerber A, Ermis H, Miny P (2002): Nackentransparenzscreening im 1. Trimenon: Ergebnisse einer prospektiven Studie bei 1980 Feten aus einem Zentrum in der Schweiz. Ultraschall in Med 23, 22-26 
The Fetal Medicine Foundation (2009): FMF Certificate of competence in the measurement of nuchal translucency, aus: Certificates of competence, 2009. URL: http://www.fetalmedicine.com/fmf/training-certification/certificates-of-competence/1113-week-scan/nuchal/ (Stand: 25.11.2009)

Tomlinson MW, Treadwell MC, Bottoms SF (1997): Isolated mild ventriculomegaly: associated karyotypic abnormalities and in utero observations. J Matern Fetal Med $\underline{6}$, 241-244

Van den Hof MC, Wilson RD, Diagnostic Imaging Committee, Society of Obstetricians and Gynaecologists of Canada, Genetics Committee (2005): Fetal soft markers in obstetric ultrasound. J Obstet Gynaecol Can 27, 592-636

Walkinshaw S, Pilling D, Spriggs A (1994): Isolated choroid plexus cysts - the need for routine offer of karyotyping. Prenat Diagn 14, 663-667

Wax JR, Cartin A, Pinette MG, Blackstone J (2005): Does the Frequency of Soft Sonographic Aneuploidy Markers Vary by Fetal Sex? J Ultrasound Med 24, 1059-1063

Wax JR, Royer D, Mather J, Chen C, Aponte-Garcia A, Steinfeld JD, Ingardia CJ (2000): A preliminary study of sonographic grading of fetal intracardiac foci: feasibility, reliability and association with aneuploidy. Ultrasound Obstet Gynecol 16, 123-127

Wickstrom EA, Thangavelu M, Parilla BV, Tamura RK, Sabbagha RE (1996): A prospective study of the association between isolated fetal pyelectasis and chromosomal abnormality. Obstet Gynecol 묘, 379-382

Winter TC, Anderson AM, Cheng EY, Komarniski CA, Souter VL, Uhrich SB, Nyberg DA (2000): Echogenic intracardiac focus in 2nd-trimester fetuses with trisomy 21: usefulness as a US marker. Radiology $\underline{216}, 450-456$ 
Wolman I, Jaffa A, Geva E, Diamant S, Strauss S, Lessing JB, Yaron Y (2000): Intracardiac echogenic focus: no apparent association with structural cardiac abnormality. Fetal Diagn Ther $\underline{15}, 216-218$ 


\section{Lebenslauf}

Am 10. August 1982 wurde ich als einzige Tochter von Frau Dr. med. Ute Knauer-Fern, geb. Fern, und Herrn Prof. Dr. med. August-Wilhelm Schmidt in Detmold geboren.

Von 1988 bis 1992 besuchte ich die Grundschule „Bachschule“ in Detmold. Im Sommer 1992 wechselte ich an das Christian-Dietrich-Grabbe-Gymnasium Detmold, wo ich zunächst die Unter- und Mittelstufe absolvierte.

Die 11. Klasse nutzte ich für ein Auslandsjahr und verbrachte das Schuljahr 1998/99 am Lancing College in Großbritannien. 1999 kehrte ich an das Christian-Dietrich-GrabbeGymnasium Detmold zurück, wo ich im Juni 2001 mein Abitur ablegte.

Im Oktober 2001 nahm ich mein Studium der Humanmedizin an der Georg-AugustUniversität Göttingen auf. Dort legte ich im September 2003 das Physikum ab. Nach dem 1. klinischen Semester, welches ich ebenfalls noch in Göttingen verbrachte, wechselte ich zum Sommersemester 2004 an die Eberhard-Karls-Universität Tübingen. Dort absolvierte ich den verbleibenden klinischen Studienabschnitt.

Im August 2006 begann ich mein Praktisches Jahr in der Abteilung für Chirurgie \& Orthopädie am Hôpital cantonal de Fribourg in der französischen Schweiz. Im Dezember 2006 kam ich zurück nach Deutschland und absolvierte die Tertiale der Inneren Medizin und meines Wahlfaches Neurologie am Marienhospital Stuttgart. Im November 2007 legte ich die Ärztliche Prüfung in Tübingen ab.

Im März 2008 trat ich eine Stelle als Assistenzärztin in der Abteilung für Innere Medizin im Augusta-Krankenhaus Düsseldorf an. Bereits im Juni 2008 wechselte ich dann in die Abteilung für Innere Medizin des Johanna-Etienne-Krankenhauses Neuss.

Nach diesen Monaten der klinischen Erfahrung wechselte ich im Dezember 2008 schließlich in die Abteilung für diagnostische \& interventionelle Radiologie des Johanna-EtienneKrankenhauses Neuss, da ich die Facharztweiterbildung im Fach Radiologie anstrebe. 


\section{Danksagung}

Mein Dank gilt Herrn Privatdozent Dr. med. Hans-Ulrich Pauer für die Vergabe dieses Promotionsthemas, für seine intensive und freundschaftliche Betreuung der Arbeit sowie seine ständige Diskussionsbereitschaft.

Danken möchte ich auch Herrn Professor Dr. med. Thomas Krauß für seine stete Hilfsbereitschaft und seine konstruktive Kritik.

Herrn Dr. med. Werner Stein danke ich für seine Hilfe bei der Sichtung der Daten sowie für die Zusammenarbeit bei der Präsentation der Studie beim Kongress der Norddeutschen Gesellschaft für Gynäkologie und Geburtshilfe (NGGG) im September 2007.

Ich danke Herrn Professor Dr. med. Rüdiger Rauskolb für seine Hilfe bei der Sichtung der Daten der Frauenklinik Northeim.

Ganz besonderer Dank gilt meiner Mutter für ihre bedingungslose Unterstützung in der Zeit meines Medizinstudiums sowie für ihre ständige Hilfsbereitschaft in jeder Phase meiner Dissertation und das sorgfältige Korrekturlesen derselben.

Sebastian danke ich für seine Hilfe bei der Auswertung der Daten, für das Korrekturlesen dieser Arbeit und für seine große Geduld und Unterstützung. 